\title{
A DIFFUSE-INTERFACE APPROACH FOR MODELING TRANSPORT, DIFFUSION AND ADSORPTION/DESORPTION OF MATERIAL QUANTITIES ON A DEFORMABLE INTERFACE*
}

\author{
KNUT ERIK TEIGEN ${ }^{\dagger}$, XIANGRONG LI ${ }^{\ddagger}$, JOHN LOWENGRUB ${ }^{\S}$, FAN WANG ${ }^{\Uparrow}$, AND \\ AXEL VOIGT"
}

\begin{abstract}
A method is presented to solve two-phase problems involving a material quantity on an interface. The interface can be advected, stretched, and change topology, and material can be adsorbed to or desorbed from it. The method is based on the use of a diffuse interface framework, which allows a simple implementation using standard finite-difference or finite-element techniques. Here, finite-difference methods on a block-structured adaptive grid are used, and the resulting equations are solved using a non-linear multigrid method. Interfacial flow with soluble surfactants is used as an example of the application of the method, and several test cases are presented demonstrating its accuracy and convergence.
\end{abstract}

Key words. Partial differential equations, diffuse interface, interfacial dynamics, complex geometry,multigrid, adaptive grid, finite difference, multiphase, adsorption, desorption

AMS subject classifications. 35Q35, 35K05, 35K57, 65Z05, 65M06, 65M50, 65M55, 76Txx, $82 \mathrm{C} 24$.

1. Introduction Many problems in the biological, physical and engineering sciences involve systems of equations that need to be solved in evolving domains with complex shapes. In addition, the solutions in the bulk domain may couple with the surface through adsorption of mass from the bulk to the surface and desorption from the surface to the bulk. Furthermore, the evolution of the domain boundary may depend on the distribution of the surface concentration through the modification of interfacial forces. Surfactants are a classic example where the amphiphilic organic compounds may adsorb to and desorb from a liquid/liquid or liquid/gas interface and lower the surface tension on the interface. Thus, inhomogeneous distribution of surfactants produces Marangoni forces - tangential forces along the interface - that affect the dynamics; surfactants play important roles in vortex pair interaction (e.g., [86, 34]), fingering (e.g., $[84,63])$ and drop break-up and coalescence (e.g., $[35,36,48,32])$. Other examples include biomembranes where transmembrane proteins play an important role in intraand extra-cellular dynamics (e.g., [46, 2, 51, 29]), epitaxially grown thin films where adsorbing/desorbing adatoms affect the dynamics and coarsening of the thin film (e.g., $[23,81,52])$, and electrochemical dissolution of binary alloys where one component is removed selectively and dissolved in an electrolyte solution (e.g., $[19,15])$.

\footnotetext{
${ }^{*}$ Received: June 4, 2009; accepted (in revised version): August 26, 2009. Communicated by Chun Liu.

${ }^{\dagger}$ Department of Energy and Process Engineering, Norwegian University of Science and Technology, 7491 Trondheim, Norway (knut.erik.teigen@ntnu.no)

${ }^{\ddagger}$ Department of Mathematics, University of California, Irvine, Irvine CA-92697, USA (xli@math.uci.edu)

$\S$ Department of Mathematics, University of California, Irvine, Irvine CA-92697, USA (lowengrb@math.uci.edu)

"Department of Mathematics, University of California, Irvine, Irvine CA-92697, USA (wangf@math.uci.edu)

" Department of Mathematics, Technische Universitt Dresden, 01062 Dresden, Germany (axel.voigt@tu-dresden.de)
} 
From a numerical point of view, solving a coupled bulk/surface system of equations on a moving, complex domain is highly challenging; the domain boundary may stretch, break-up or coalesce with other interfaces. Adsorption of mass to, and desorption of mass from, the interfaces poses another challenge. Furthermore, the surface concentration may only be soluble in either the exterior or interior of the domain (e.g., amphiphilic nature of surfactants). The available numerical methods for solving these problems can roughly be divided into two categories: interface tracking and interface capturing methods. Interface tracking methods use either through a separate grid for the interface, or a set of interconnected points to mark the interface. For example, boundary integral methods use a surface mesh to track the interface. In the context of surfactants, a boundary integral method for studying the effect of insoluble surfactants on drop deformation was developed in [82]. This method was extended to arbitrary viscosity ratios in [67], and to soluble surfactants in [66]. Another tracking method is the front-tracking method, where a fixed grid is used to compute the flow, while a set of connected marker particles is used to track the interface and any interfacial quantities. A front-tracking method for insoluble surfactants was developed in [39], and this method was extended to handle soluble surfactants in [91] and [69]. Lagrangian approaches are typically very accurate, but can be relatively complicated to implement, especially in three dimensions and for problems involving topological changes.

In interface capturing methods, the interface is not tracked explicitly, but instead is implicitly defined through a regularization of the interface. This means that the solution of the problem can be done independently of the underlying grid, which greatly simplifies gridding, discretization, and handling of topological changes. For example, a volume-of-fluid (VOF) method for insoluble surfactants was developed in [75]. A more general method which allows non-linear equations of state for surface tension was then developed in [38]. A level-set method for solving the surfactant equation was presented in [89], and later coupled to an external flow solver in [88]. An alternative approach tracking and approach was developed in [90, 32], using the socalled Arbitrary Lagrangian-Eulerian (ALE) method. An immersed interface boundary method for interfacial flows with insoluble surfactants was recently developed in [47]. In the context of thin films, a level-set method for the simulating the motion of thin films under surface diffusion with free adatoms was developed in [81]. In addition, an immersed interface method was developed to simulate electrodeposition in an evolving complex domain [77]. Level-set methods for solving more general equations on implicitly defined, but stationary, surfaces have also been developed in $[5,30]$.

Other approaches for solving equations in complex domains include fictitious domain methods (e.g., [27, 28, 65, 31, 70, 12, 74, 72, 56, 33]), immersed interface methods (e.g., [49, 54, 37]), modified finite volume/embedded boundary/cut-cell methods (e.g., [41, 64, 62, 40, 42, 76, 59]) and ghost fluid methods (e.g., [20, 24, 25, $26,60,61])$. All these methods, however, require non-standard tools typically not available in standard finite element and finite difference software packages.

The diffuse-interface, or phase-field, method represents yet another approach for simulating solutions of equations in complex, evolving domains. In this method, which we follow here, the complex domain is represented implicitly by a phase-field function, which is an approximation of the characteristic function of the domain. The domain boundary is replaced by a narrow diffuse interface layer such that the phase-field function rapidly transitions from one inside the domain to zero in the exterior of the domain. The boundary of the domain can thus be represented as an isosurface of 
the phase-field function. The bulk and surface PDEs are then extended on a larger, regular domain with additional terms that approximate the adsorption-desorption flux boundary conditions and source terms for the bulk and surface equations respectively. Standard finite-difference or finite-element methods may be used. Here, we focus on a finite difference approach.

The diffuse interface method, which has a long history in the theory of phase transitions dating back to van der Waals (e.g., [79, 3]), was used in [46] to study diffusion inside a cell with zero Neumann boundary conditions at the (stationary) cell-boundary (see also $[8,9]$ ), and later was used to simulate electrical waves in the heart [21]. This approach has been extended [51] to simulate coupled bulk diffusion with an ordinary-differential equation description of reaction-kinetics on the bounding surface of a stationary domain to simulate membrane-bound Turing patterns. More recently, general diffuse-interface methods have been developed for solving PDEs on stationary surfaces [73], evolving surfaces [13, 14, 16, 17] and for solving PDEs in complex evolving domains with Dirichlet, Neumann and Robin boundary conditions [53].

As shown in the previous paragraphs, bulk/surface problems are important in a wide range of areas. Here, we combine and refine previous work on diffuse-interface methods to develop a new method for solving coupled bulk/surface problems on general, evolving domains. The method is very simple compared to other methods, and can handle advection, diffusion and adsorption/desorption in a straight-forward manner. Matched asymptotic expansions are used to demonstrate that the diffuse interface system converges to the original sharp interface equations as the interface thickness tends to zero. The use of a non-linear multigrid method and block-structured, adaptive grids also make the method computationally efficient. We present several test cases demonstrating the accuracy and convergence of the proposed method.

The paper is organized as follows. In section 2, the governing equations for the surface concentration and the bulk concentration are introduced, and the interface representation presented. Section 3 presents an asymptotic analysis of the proposed method. Section 4 then details the numerical implementation. In section 5 , the performance of the numerical method is evaluated on a set of test cases. Finally, section 6 contains conclusions and discussions of future work.

\section{Mathematical formulation}

2.1. Governing equations Consider a domain $\Omega \subset \mathbb{R}^{2,3}$, which contains a closed interface, $\Gamma$. The interior of the interface is $\Omega_{0} \subset \Omega$, and the exterior is $\Omega_{1} \subset \Omega$. See figure 2.1 for an illustration. Let $f$ denote a surface concentration defined on $\Gamma$. We suppose that $f$ is extended off $\Gamma$ constant in the normal direction (i.e., $\boldsymbol{\nabla} f \cdot \mathbf{n}=0$ ) so that $f$ is defined in a neighborhood of $\Gamma$. Then, the sharp interface mass conservation equation is

$$
\frac{\partial f}{\partial t}+\mathbf{u} \cdot \boldsymbol{\nabla} f=\boldsymbol{\nabla}_{\Gamma} \cdot\left(D_{f} \boldsymbol{\nabla}_{\Gamma} f\right)-f \boldsymbol{\nabla}_{\Gamma} \cdot \mathbf{u}_{\Gamma}-f \kappa u_{n}+j,
$$

where $\mathbf{u}$ is the velocity, $\boldsymbol{\nabla}_{\Gamma}=(\mathbf{I}-\mathbf{n n}) \boldsymbol{\nabla}$ is the surface gradient, $\mathbf{n}$ is the normal vector to $\Gamma$ pointing into $\Omega_{0}, D_{f}$ is the diffusion coefficient, $\mathbf{u}_{\Gamma}=(\mathbf{I}-\mathbf{n n}) \mathbf{u}, u_{n}=\mathbf{u} \cdot \mathbf{n}, \kappa$ is the total curvature of $\Gamma$ (positive for a sphere), and $j$ is a source term that arises from adsorption to and desorption from $\Gamma$

$$
j=r_{a} F-r_{d} f,
$$




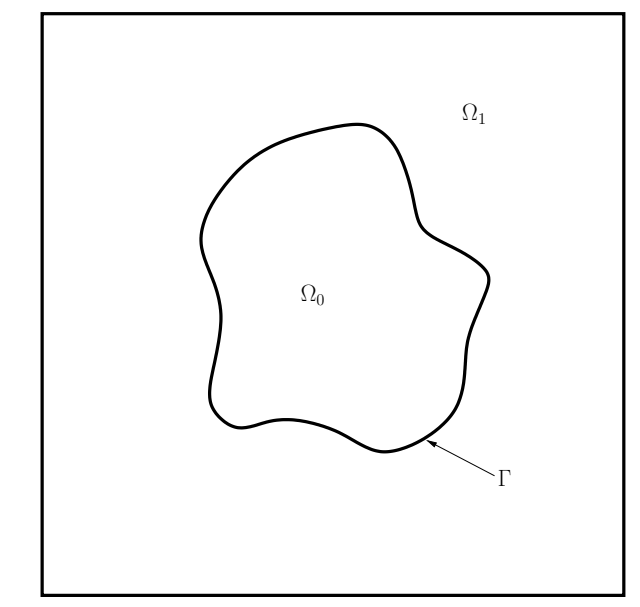

Fig. 2.1: Illustration of the mathematical domain.

where $r_{a}$ and $r_{d}$ are adsorption and desorption coefficients, respectively, and $F$ is the bulk concentration (evaluated immediately adjacent to $\Gamma$ ). Note that in the context of surfactants, the interface may become saturated and instead one may use

$$
j=r_{a} F\left(f_{\infty}-f\right)-r_{d} f,
$$

where $f_{\infty}$ is the maximum interface concentration. An equivalent formulation is

$$
\frac{\partial f}{\partial t}+\nabla_{\Gamma} \cdot(\mathbf{u} f)=\nabla_{\Gamma} \cdot\left(D_{f} \nabla_{\Gamma} f\right)+j .
$$

We refer the reader also to [11] for further discussion of formulations involving interfacial transport using constant normal extensions and to [38] for Eulerian formulations of the dynamics of surface concentrations.

Assume that the surface concentration $f$ is soluble in $\Omega_{1}$, but not in $\Omega_{0}$. Then, define the bulk concentration in $\Omega_{1}$ to be $F$, which evolves according to the bulk mass conservation equation

$$
\frac{\partial F}{\partial t}+\boldsymbol{\nabla} \cdot(F \mathbf{u})=D_{F} \nabla^{2} F \text { in } \Omega_{1},
$$

with the boundary condition at $\Gamma$

$$
D_{F} \boldsymbol{\nabla} F \cdot \mathbf{n}=-j \text { on } \Gamma \text {. }
$$

Note that if the surface concentration were soluble in $\Omega_{0}$, then an additional mass conservation equation would need to be posed. Our formulation is sufficiently general to handle this case.

Next, we consider a distribution formulation of equation (2.4) by introducing a surface delta function $\delta_{\Gamma}$, such that

$$
\int_{\Gamma} f \mathrm{~d} \Gamma=\int_{\Omega} f \delta_{\Gamma} \mathrm{d} \Omega
$$


where $\Omega=\Omega_{1} \cup \Omega_{0}$ (actually the above equation holds for any domain $\Omega$ that contains $\Gamma)$. The mass conservation equation may be rewritten accordingly as

$$
\frac{\partial}{\partial t}\left(f \delta_{\Gamma}\right)+\nabla \cdot\left(f \delta_{\Gamma} \mathbf{u}\right)=\boldsymbol{\nabla} \cdot\left(\delta_{\Gamma} D_{f} \boldsymbol{\nabla} f\right)+\delta_{\Gamma} j .
$$

This distribution formulation formally holds in $\Omega$.

Analogously, the bulk equation (2.5) may be extended to hold in $\Omega$ in distribution form. Introducing the Heaviside function

$$
H=\left\{\begin{array}{l}
1 \text { in } \Omega_{1}, \\
0 \text { in } \Omega_{0},
\end{array}\right.
$$

the bulk concentration equation (2.5) and boundary condition (2.6) may be reformulated as

$$
\frac{\partial}{\partial t}(H F)+\boldsymbol{\nabla} \cdot(H F \mathbf{u})=D_{F} \boldsymbol{\nabla} \cdot(H \nabla F)-\delta_{\Gamma} j,
$$

where the boundary condition has been included as a singular source term following [53].

2.2. Interface representation A phase-field function $c$ may be used to approximate the characteristic function of $\Omega_{1}$. Let

$$
c(\mathbf{x}, t)=\frac{1}{2}\left[1+\tanh \left(\frac{r(\mathbf{x}, t)}{2 \sqrt{2} \epsilon}\right)\right],
$$

where $\epsilon$ is a small parameter related to the interface thickness and $r(\mathbf{x}, t)$ is a signed distance function to $\Gamma$ (positive in $\Omega_{1}$ ). The position of the interface may be taken to be $\Gamma(t)=\{\mathbf{x} \in \Omega \mid c(\mathbf{x}, t)=1 / 2\}$. To evolve $c$, one may evolve $r$ by

$$
\frac{\partial r}{\partial t}+\mathbf{v} \cdot \nabla r=0
$$

where $\mathbf{v}$ is an extension of $\mathbf{u}$ off the interface which is constant in the normal direction [1]. Alternatively, an advective Cahn-Hilliard equation can be used,

$$
\begin{aligned}
\frac{\partial c}{\partial t}+\mathbf{u} \cdot \boldsymbol{\nabla} c & =\boldsymbol{\nabla} \cdot(M(c) \boldsymbol{\nabla} \mu), \\
\mu & =g^{\prime}(c)-\epsilon^{2} \nabla^{2} c,
\end{aligned}
$$

where $\mu$ is a chemical potential. Here, we take $g(c)=\frac{1}{4} c^{2}(1-c)^{2}$ as the double well potential. Note that this polynomial energy does not constrain $c \in[0,1]$ but the deviation from this interval is typically $O(\epsilon)$ at most. Alternative choices (e.g., doubleobstacle $[6,7]$ or logarithmic potentials [4]) may be used that do constrain $c \in[0,1]$. The mobility $M$ is localized on the interface and is taken to be $M(c)=\sqrt{4 g(c)}$. This equation is fourth-order and nonlinear and thus requires specialized numerical methods to solve in an efficient manner. This is discussed further in section 4.

2.3. Regularized delta and Heaviside functions In order to evaluate equation (2.8) and equation (2.10) numerically, regularizations of the surface delta function and Heaviside function are needed. In the phase-field context, several definitions of 
the delta function are available from the literature. In this work, the approximation from [73],

$$
\delta_{\Gamma} \approx \frac{3 \sqrt{2}}{\epsilon} B(c), \quad B(c)=c^{2}(1-c)^{2},
$$

is used for the surface equation. Note that other choices may be used [17]. For the boundary condition in the bulk equation, the approximation

$$
\delta_{\Gamma} \approx|\nabla c|
$$

is used, which avoids additional scaling issues in the equation [53]. Further, the regularized Heaviside function is simply taken to be [53]

$$
H(c) \approx c .
$$

The final system of equations can now be summarized as

$$
\begin{aligned}
\frac{\partial}{\partial t}(B(c) f)+\boldsymbol{\nabla} \cdot(B(c) f \mathbf{u}) & =\boldsymbol{\nabla} \cdot\left(D_{f} B(c) \boldsymbol{\nabla} f\right)+B(c) j, \\
\frac{\partial}{\partial t}(c F)+\boldsymbol{\nabla} \cdot(c F \mathbf{u}) & =D_{F} \boldsymbol{\nabla} \cdot(c \boldsymbol{\nabla} F)-|\boldsymbol{\nabla} c| j .
\end{aligned}
$$

3. Asymptotic analysis In this section, the method of matched asymptotic expansions is used to provide a formal justification for the diffuse interface approach. To make the system slightly more general, we may add reaction terms to the bulk and surface equations, i.e., $R_{f}(f)$ and $R_{F}(F)$ may be added to equations (2.18) and (2.19) respectively. In this approach, the domain $\Omega$ is separated into two regions the regions far from $\Gamma$ (outer region, i.e., the portions of $\Omega_{1}$ and $\Omega_{0}$ away from $\Gamma$ ) and the region near $\Gamma$ (inner region). In each region, the variables are expanded in powers of the diffuse interface thickness. In the outer region, the variables are expanded as

$$
c(\mathbf{x}, t)=c_{0}(\mathbf{x}, t)+\epsilon c_{1}(\mathbf{x}, t)+\ldots,
$$

and analogously for the other variables. In the region near $\Gamma$, we introduce a new coordinate system.

3.1. New Coordinate Introduce the following local normal-tangential coordinate system with respect to the curve $\Gamma$. Let $\Gamma=\mathbf{X}(\mathbf{s}, t)=(X(\mathbf{s}, t), Y(\mathbf{s}, t), Z(\mathbf{s}, t))$, where $\mathbf{s}=\left(s_{1}, s_{2}\right)$ is a parametrization of the surface and $t$ is time. Let $r=r(\mathbf{x} ; \epsilon)$ be the signed distance along the normal from a point $\mathbf{x}$ to $\Gamma$, and is positive when outside $\Gamma$ (i.e. in $\Omega_{1}$ ). Then, if $\Gamma$ is smooth, there exists a neighborhood

$$
U_{\epsilon}:=\{\mathbf{x} \in \Omega:|r(\mathbf{x}, \epsilon)|<\rho\}
$$

of $\Gamma$ for some $0<\rho \ll 1$, such that the local coordinate transformation from $(x, y, z)$ to $\left(r, s_{1}, s_{2}\right)$ is valid, e.g., $\mathbf{x}=\mathbf{X}(\mathbf{s}, t)+r \mathbf{n}(\mathbf{s}, t)$. Near the interface, we introduced a stretched normal coordinate $z=\frac{r}{\epsilon}$. Note that as $\epsilon \rightarrow 0$, the inner region extends from $-\infty<z<\infty$. We then assume that the variables may be expanded in regular power series in $\epsilon$ in the stretched coordinate system:

$$
c(\mathbf{x}, t)=C(z, \mathbf{s}, t)=C_{0}(z, \mathbf{s}, t)+\epsilon C_{1}(z, \mathbf{s}, t)+\ldots,
$$


and analogously for the other variables. Furthermore, in this coordinate system we have

$$
\begin{gathered}
\partial_{t}=-\epsilon^{-1} \hat{V}_{0} \partial_{z}+\partial_{t}+\mathcal{O}(\epsilon), \\
\boldsymbol{\nabla}=\epsilon^{-1} \mathbf{n} \partial_{z}+\nabla_{\Gamma}+\mathcal{O}(\epsilon),
\end{gathered}
$$

where $\hat{V}_{0}$ is the leading term of the normal velocity of $\Gamma$.

3.2. Matching Condition Assuming that there is an overlapping region where both the inner and outer expansions are valid, we may write the outer expansion in the local coordinate system as

$$
c(\mathbf{x}, t)=c(r, \mathbf{s}, t)=c_{0}(r, \mathbf{s}, t)+\epsilon c_{1}(r, \mathbf{s}, t)+\ldots,
$$

and analogously for the other variables. Matching the inner and outer expansions in this region, the following matching conditions hold [10,71, 22]

$$
\begin{aligned}
\lim _{r \rightarrow 0^{ \pm}} F_{0} & =\lim _{z \rightarrow \pm \infty} \hat{F}_{0}, \\
\lim _{r \rightarrow 0^{ \pm}} f_{0} & =\lim _{z \rightarrow \pm \infty} \hat{f}_{0}, \\
\lim _{r \rightarrow 0 \pm} \mathbf{n} \cdot \nabla F_{0} & =\lim _{z \rightarrow \pm \infty} \partial_{z} \hat{F}_{1}, \\
\lim _{z \rightarrow \pm \infty} \partial_{z} \hat{F}_{0} & =0 .
\end{aligned}
$$

Analogous matching conditions hold for the other variables.

\subsection{Bulk Equation}

3.3.1. Outer expansion The $\mathcal{O}\left(\epsilon^{0}\right)$ term of equation (2.19) gives:

$$
\frac{\partial F_{0}}{\partial t}+\boldsymbol{\nabla} \cdot\left(F_{0} \mathbf{u}_{0}\right)=\boldsymbol{\nabla} \cdot\left(D_{F} \boldsymbol{\nabla} F_{0}\right)+R_{F}\left(F_{0}\right) .
$$

Thus at leading order equation (2.5), with the reaction term, is recovered. To determine the boundary conditions on $\Gamma$, we match with the inner expansion.

3.3.2. Inner expansion At $\mathcal{O}\left(\epsilon^{-2}\right)$, we obtain

$$
\partial_{z}\left(C_{0} D_{F} \partial_{z} \hat{F}_{0}\right)=0
$$

which implies $\partial_{z} \hat{F}_{0}=0$. At $\mathcal{O}\left(\epsilon^{-1}\right)$ we obtain

$$
\left(U_{N, 0}-\hat{V}_{0}\right) \partial_{z}\left(C_{0} \hat{F}_{0}\right)=\partial_{z}\left(C_{0} D_{F} \partial_{z} \hat{F}_{1}\right)+\left(r_{a} \hat{F}_{0}-r_{d} \hat{f}_{0}\right) \partial_{z} C_{0}
$$

Since $U_{N, 0}=\hat{V}_{0}$ by the asymptotic analysis of the advective Cahn-Hilliard equation [58], $\hat{F}_{0}$ is independent of $z$ and, as we show below, $\hat{f}_{0}$ is also independent of $z$, we may integrate equation (3.12) and use that $\int_{-\infty}^{+\infty} \partial_{z} C_{0} d z=1$, again taken from [58], to obtain

$$
D_{F} \lim _{z \rightarrow+\infty} \partial_{z} \hat{F}_{1}=-\left(r_{a} \hat{F}_{0}-r_{d} \hat{f}_{0}\right) .
$$

Together with equation (3.8), we therefore recover the Neumann boundary condition (2.6) for the outer solution at leading order

$$
D_{F} \lim _{r \rightarrow 0+} \mathbf{n} \cdot \nabla F_{0}=D_{F} \lim _{z \rightarrow+\infty} \partial_{z} \hat{F}_{1}=-\left(r_{a} F_{0}-r_{d} f_{0}\right),
$$

where we have set $\hat{f}_{0}=f_{0}$ since $\hat{f}_{0}$ is independent of $z$. Next, we turn to the surface concentration equation. 
3.4. Surface Equation Here, we only focus on the inner expansion since the equation is localized around the interface. At $\mathcal{O}\left(\epsilon^{-2}\right)$, we obtain

$$
\partial_{z}\left(D_{f} B\left(C_{0}\right) \partial_{z} \hat{f}_{0}\right)=0
$$

which implies $\partial_{z} \hat{f}_{0}=0$, as claimed above. The $\mathcal{O}\left(\epsilon^{-1}\right)$ term gives

$$
0=\partial_{z}\left(B\left(C_{0}\right) \partial_{z} \hat{f}_{1}\right)
$$

where we have used that $U_{N, 0}=\hat{V}_{0}$. Equation (3.16) implies that $\partial_{z} \hat{f}_{1}=0$ also. At $\mathcal{O}\left(\epsilon^{0}\right)$, we obtain

$$
\begin{aligned}
\partial_{t}\left(B\left(C_{0}\right) \hat{f}_{0}\right)+\nabla_{\Gamma} \cdot\left(B\left(C_{0}\right) \hat{f}_{0} \mathbf{U}_{0}\right)=\partial_{z} & \left(D_{f} B\left(C_{0}\right) \partial_{z} \hat{f}_{2}\right)+\nabla_{\Gamma} \cdot\left(D_{f} B\left(C_{0}\right) \nabla_{\Gamma} \hat{f}_{0}\right) \\
& +B\left(C_{0}\right)\left(r_{a} \hat{F}_{0}-r_{d} \hat{f}_{0}\right)+B\left(C_{0}\right) R_{f}\left(f_{0}\right) .
\end{aligned}
$$

Since $\hat{f}_{0}$ is independent of $z$, we may integrate equation (3.17) in z from $-\infty$ to $+\infty$, and divide by $\int_{-\infty}^{+\infty} B\left(C_{0}\right) \mathrm{d} z>0$ to obtain

$$
\partial_{t} f_{0}+\nabla_{\Gamma} \cdot\left(f_{0} \mathbf{u}_{0}\right)=\nabla_{\Gamma} \cdot\left(D_{f} \nabla_{\Gamma} f_{0}\right)=r_{a} F_{0}-r_{d} f_{0}+R_{f}\left(f_{0}\right)
$$

where we taken $\hat{f}_{0}=f_{0}, \hat{F}_{0}=F_{0}$ and we also have assumed that $\mathbf{U}_{\Gamma, 0}$ is independent of $z$ (which implies there is no jump in velocity across $\Gamma$ ) so that we may write $\mathbf{U}_{0}=\mathbf{u}_{0}$. Thus, equation (2.4), with the reaction term, is recovered at leading order.

4. Numerical methods This section briefly describes the numerical methods used to solve the above equations. The algorithm follows the one developed in [87]. In particular, the equations are discretized using finite differences in space and a semiimplicit time discretization. A block-structured, adaptive grid is used to increase the resolution around the interface in an efficient manner. The nonlinear equations at the implicit time level are solved using a non-linear Adaptive Full Approximation Scheme (AFAS) multigrid algorithm. For a detailed discussion of the adaptive algorithm and the multigrid solver, the reader is referred to [87].

The equations are discretized on a rectangular domain. The surface concentration, the bulk concentration, the phase-field function and the chemical potential are defined at the cell-centers, while the velocity components are defined on cell-edges.

Special care has to be taken for the temporal discretization. The Cahn-Hilliard system is fourth order in space, and requires the use of an implicit method to avoid severe limitations in the time step. Here, Crank-Nicholson type schemes are used [45],

$$
\begin{aligned}
\frac{c^{k+1}-c^{k}}{\Delta t}= & -\frac{1}{2}\left[\boldsymbol{\nabla}_{d} \cdot\left(\mathbf{u}^{k+1} c^{k+1}\right)+\boldsymbol{\nabla}_{d} \cdot\left(\mathbf{u}^{k} c^{k}\right)\right] \\
& +\frac{1}{2}\left[\boldsymbol{\nabla}_{d} \cdot\left(M^{k+1} \boldsymbol{\nabla}_{d} \mu^{k+1}\right)+\boldsymbol{\nabla}_{d} \cdot\left(M^{k} \boldsymbol{\nabla}_{d} \mu^{k}\right)\right], \\
\mu^{k+1}= & g^{\prime}\left(c^{k+1}\right)-\epsilon^{2} \boldsymbol{\nabla}_{d}^{2} c^{k+1} .
\end{aligned}
$$

In $[45,87]$ this approach was shown to be robust and efficient. The equations for 
surface concentration and bulk concentration are discretized in a similar fashion,

$$
\begin{aligned}
\frac{\delta_{\Gamma}^{k+1} f^{k+1}-\delta_{\Gamma}^{k} f^{k}}{\Delta t}= & -\frac{1}{2}\left[\boldsymbol{\nabla}_{d} \cdot\left(\mathbf{u}^{k+1} \delta_{\Gamma}^{k+1} f^{k+1}\right)+\boldsymbol{\nabla}_{d} \cdot\left(\mathbf{u}^{k} \delta_{\Gamma}^{k} f^{k}\right)\right] \\
& +\frac{D_{f}}{2}\left[\boldsymbol{\nabla}_{d} \cdot\left(\delta_{\Gamma}^{k+1} \boldsymbol{\nabla}_{d} f^{k+1}\right)+\boldsymbol{\nabla}_{d} \cdot\left(\delta_{\Gamma}^{k} \boldsymbol{\nabla}_{d} f^{k}\right)\right] \\
& +\frac{1}{2}\left[\delta_{\Gamma}^{k+1} j^{k+1}+\delta_{\Gamma}^{k} j^{k}\right], \\
\frac{H^{k+1} F^{k+1}-H^{k} F^{k}}{\Delta t}=- & \frac{1}{2}\left[\boldsymbol{\nabla}_{d} \cdot\left(\mathbf{u}^{k+1} H^{k+1} F^{k+1}\right)+\nabla_{d} \cdot\left(\mathbf{u}^{k} H^{k} F^{k}\right)\right] \\
& +\frac{D_{f}}{2}\left[\boldsymbol{\nabla}_{d} \cdot\left(H^{k+1} \boldsymbol{\nabla}_{d} F^{k+1}\right)+\boldsymbol{\nabla}_{d} \cdot\left(H^{k} \boldsymbol{\nabla}_{d} F^{k}\right)\right] \\
& +\frac{1}{2}\left[\left|\nabla_{d} c^{k+1}\right| j^{k+1}+\left|\nabla_{d} c^{k}\right| j^{k}\right],
\end{aligned}
$$

where, in the above equations, we set $\delta_{\Gamma}^{k+1}=B\left(c^{k+1}\right)+\alpha$ and $H^{k+1}=\sqrt{\left(c^{k+1}\right)^{2}+\alpha^{2}}$, with $\alpha$ being a small parameter $\left(\alpha=10^{-6}\right)$ that is used to ensure that division by zero does not occur; the results are found to be quite insensitive to the precise choice of $\alpha$ provided it is sufficiently small. The operator $\nabla_{d}$ represents the standard secondorder finite-difference discretization. The convective terms of the form $\boldsymbol{\nabla} \cdot(\mathbf{u} \phi)$ are discretized using the third-order WENO reconstruction method [78, 55]. The WENO reconstruction method has the advantage that it handles steep gradients well, which may occur in the type of dynamics described in this work. Additionally, fewer grid points are needed to achieve a high order solution. This is particularly important for the efficiency of the adaptive grid, because fewer ghost cell values have to be calculated at the boundaries of each grid block.

Homogeneous Neumann far-field boundary conditions are prescribed for all variables. This is imposed by introducing a set of ghost cells around the domain. These ghost cells are updated before every smoothing operation.

The AFAS multigrid algorithm is used to solve the discretized equations at every time step. The full description of the AFAS multigrid method will not be given here, the details can be found in [87] and in the reference text [85]. The ideal run-time complexity of this algorithm is optimal, i.e., $\mathcal{O}(N)$ where $N$ is the number of grid points. The present implementation achieves this complexity, which is shown in section 5.6.

\section{Code validation}

5.1. Surface diffusion on a stationary circle First, a problem without bulk concentration is considered. This tests the validity of the diffuse interface representation of the surface equation, and of the correct implementation of the diffusion term.

Consider a stationary circle of radius $R$, with an initial surface concentration given by

$$
f_{0}(\theta)=\frac{1}{2}(1-\cos \theta)
$$

where $\theta$ denotes the angle measured in the counter-clockwise direction from the $y$-axis. The surface concentration equation can now be written in polar coordinates as

$$
\frac{\partial f(\theta, t)}{\partial t}=\frac{D_{f}}{R^{2}} \frac{\partial^{2} f(\theta, t)}{\partial \theta^{2}}
$$


with initial condition $f_{0}$ and periodic boundary condition in the $\theta$-direction. This equation can be solved analytically, yielding

$$
f(\theta, t)=\frac{1}{2}\left(1-e^{-\frac{D_{f}}{R^{2}} t} \cos \theta\right) .
$$

A series of simulations are performed comparing the numerical solution to the analytical solution. The computational domain chosen for the simulations is $[-2,2] \times$ $[-2,2]$ and a circle with radius $R=1$ is placed in the center of the domain. The phase-field function is initialized by

$$
c(x, y)=\frac{1}{2}\left[1-\tanh \left(\frac{\sqrt{x^{2}+y^{2}}-R}{2 \sqrt{2} \epsilon}\right)\right] .
$$

The initial surface concentration is given by the analytical solution, and the time step is $\Delta t=1 \times 10^{-2}$.

In the numerical code, the surface concentration is defined at grid points near the interface. To enable a direct comparison with the analytical solution, the concentration at the interface is needed. This is done by using a marching squares algorithm (see e.g. [57]) to generate a set of points at the 0.5 isocontour of the phase-field function. Bilinear interpolation is then used to interpolate the grid values of the surface concentration to these interface points. Note that this will introduce extra uncertainties, so the absolute errors given later may not be exact values. The order of convergence results should not be affected by this.

An example of the adaptive grid is shown in figure 5.1. Clearly, the grid follows the circle shape very well. No significant difference between the solutions on adaptive grids and uniform grids was found for this test case for the same effective resolution. Figure 5.2 shows comparisons between the numerical solution and the analytical solution at various times and surface diffusion coefficients. Good agreement is observed. The error in the infinity norm between the interpolated values and the exact values at the interface is given in Table 5.1. The numerical solution converges towards the exact solution in a first order fashion, as predicted by the asymptotic analysis.

Table 5.1: The error and convergence order for the surface diffusion test case at $t=1$. The interface thickness is defined as $\epsilon=1.6 h_{\min }$, where $h_{\min }$ the minimum grid size.

\begin{tabular}{ccc}
\hline Grid spacing & $\begin{array}{c}\text { Error } \\
\left(\times 10^{-2}\right)\end{array}$ & Order \\
\hline $1 / 8$ & 4.29 & - \\
$1 / 16$ & 2.35 & 0.87 \\
$1 / 32$ & 1.18 & 0.99 \\
$1 / 64$ & 0.60 & 0.98 \\
$1 / 128$ & 0.30 & 1.00 \\
\hline
\end{tabular}

5.2. Surface diffusion on an advected circle Now, the circle in the above test case is put in a constant velocity field, $\mathbf{u}=(2,0)$. The analytical solution is the same, only translated in the computational domain. The computational domain is extended by two in the $x$-direction to accomodate the translation. 


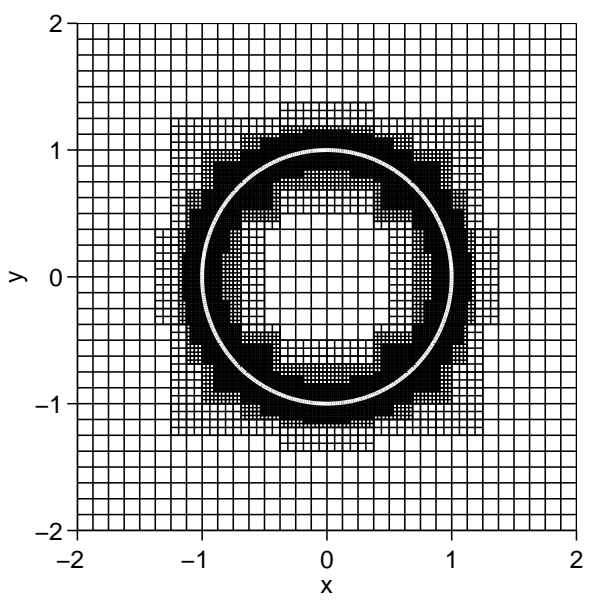

Fig. 5.1: Example of the adaptive grid for the surface diffusion on a fixed circle. The root level has $32 \times 32$ grid points and there are three levels of refinement, giving an effective resolution at the interface of $256 \times 256$.

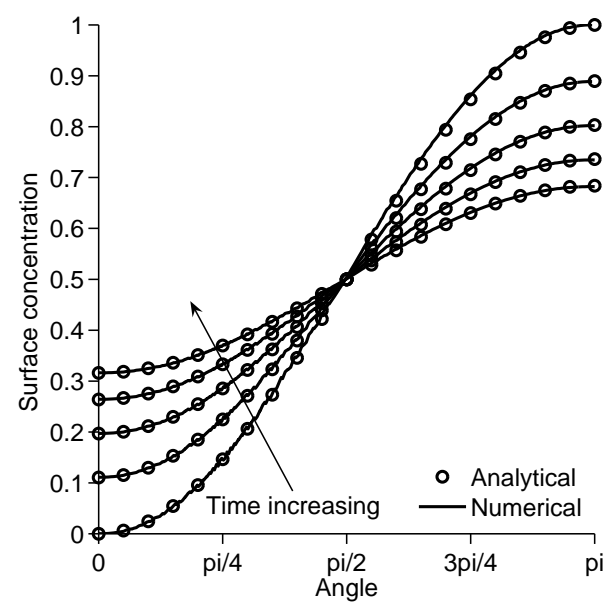

(a) Surface concentration profiles at times $t=$ $0.0,0.25,0.5,0.75$ and 1 for $D_{f}=1$.

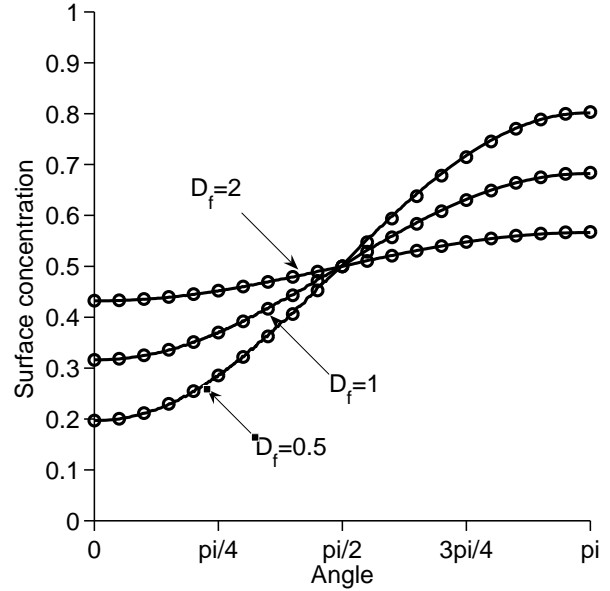

(b) Surface concentration profiles at $t=1$ for different $D_{f}$.

Fig. 5.2: Comparison of the numerical and exact solutions for the surface diffusion test case. Because the solution is symmetric with respect to the $y$-axis, only one half of the circle is shown here. The grid spacing is $h_{\min }=1 / 64$.

The initial and final surface concentration is given in figure 5.3. As shown in Table 5.2, the convergence is first order as in the diffusion only test case. The error is slightly higher due to the additional errors caused by the advection. 
Table 5.2: The error and convergence order for the diffusion on a translating circle at $t=1$. The interface thickness is defined as $\epsilon=1.6 h_{\min }$.

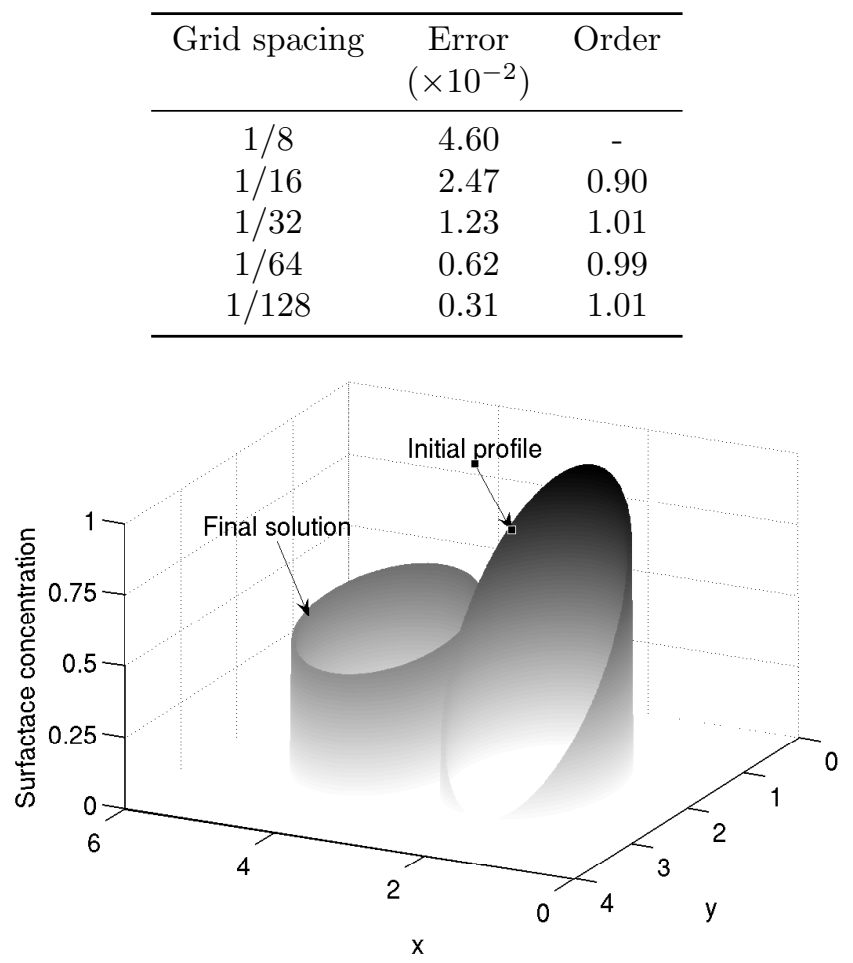

Fig. 5.3: The initial and final surface concentration for the advected circle test case.

5.3. Surface concentration evolution on an expanding circle A circle is placed in the velocity field given by

$$
u=\cos \theta, v=\sin \theta,
$$

which is illustrated in figure 5.4(a). In the absence of diffusion, the surface concentration is just a function of the circle circumference, so the mass conservation equation reduces to

$$
f(t)=\frac{C_{0}}{C(t)} f_{0},
$$

where $C=2 \pi R$ is the surface area, and subscript 0 denotes the initial condition.

For this test case, the computational domain was set to $\Omega=[-16,16] \times[-16,16]$ to accomodate the expansion. The initial radius was set to $R_{0}=1$, and the initial surface concentration was set to $f_{0}=0.5$. Instead of solving the Cahn-Hilliard system, equations (2.13)-(2.14), the phase-field function is evolved analytically for this test case. No significant difference is observed if the interface is evolved according to the Cahn-Hilliard system, as is done below. 


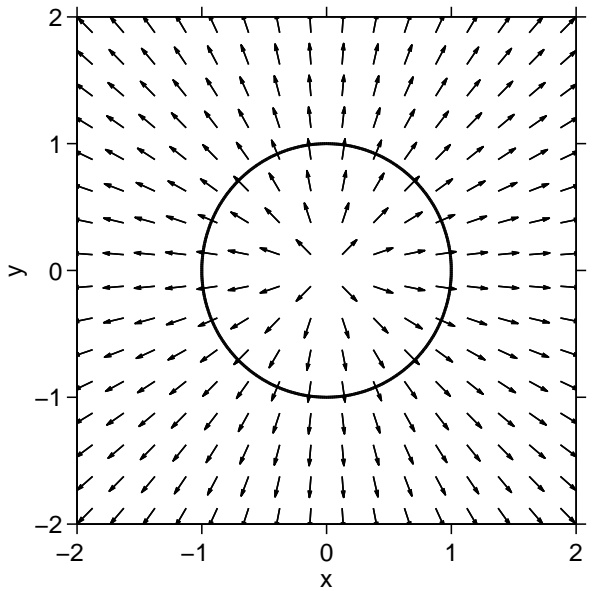

(a) Illustration of the expanding circle test case.

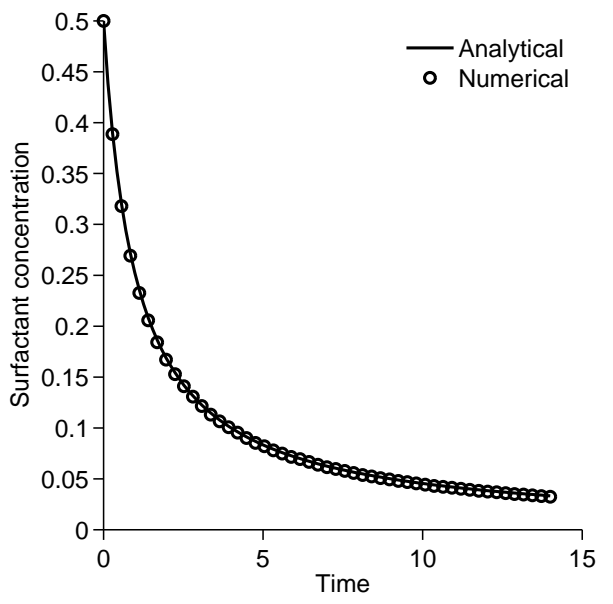

(b) Comparison of the mean value of the computed surface concentration and the analytical value. $h=1 / 8$.

Fig. 5.4: Numerical setup and results for the expanding circle test case.

The numerical and analytical solutions are compared in figure 5.4(b), and excellent agreement is observed. The convergence behaviour is given in Table 5.3, and as in previous test cases, the method converges in a first-order fashion.

Table 5.3: The error and convergence order for the expanding circle test case. The error is measured as the maximum deviation of the mean surface concentration to the analytical concentration. The interface thickness $\epsilon=1.6 h_{\min }$.

\begin{tabular}{ccc}
\hline Grid spacing & $\begin{array}{c}\text { Error } \\
\left(\times 10^{-2}\right)\end{array}$ & Order \\
\hline 1 & 13.6 & - \\
$1 / 2$ & 6.60 & 1.04 \\
$1 / 4$ & 3.30 & 1.00 \\
$1 / 8$ & 1.62 & 1.03 \\
\hline
\end{tabular}

5.4. Bulk diffusion and bulk-surface mass transfer This test case was introduced in [69] in the context of surfactants, to test the coupling between a bulk concentration and a surface concentration. Consider an initially clean circle of radius $a$ in a domain which contains an initial bulk concentration $F_{\infty}$. A simplified version of the source term is used, where mass moves from the bulk to the interface,

$$
j=r_{a} F .
$$


Table 5.4: Values used in the bulk diffusion and bulk-surface mass transfer test case.

\begin{tabular}{ll}
\hline Quantity & Value \\
\hline Radius, $a$ & 1.0 \\
Adsorption rate, $r_{a}$ & 1.0 \\
Diffusion coefficient, $D_{F}$ & 1.0 \\
Initial bulk concentration, $F_{\infty}$ & 1.0 \\
\hline
\end{tabular}

The evolution of the bulk concentration can now be described with the heat equation in an infinite, hollow cylinder,

$$
\frac{\partial F}{\partial t}=D_{F}\left(\frac{\partial^{2} F}{\partial r^{2}}+\frac{1}{r} \frac{\partial F}{\partial r}\right),
$$

with boundary conditions

$$
\begin{aligned}
& \left.\frac{\partial F}{\partial r}\right|_{r=a}=r_{a} F(r=a), \\
& F(r=R)=F_{\infty},
\end{aligned}
$$

where $R$ is the extent of the domain; $R$ is taken to be 4 . In lieu of an analytical solution, a fourth-order accurate finite-difference discretization of the above 1D problem is used for comparison to the diffuse-interface solution. The grid size for this problem was chosen high enough to give a resolution independent solution. The physical properties used in the simulations are given in Table 5.4.

A visual comparison between the $1 \mathrm{D}$ solution and the diffuse-interface results for the bulk concentration at various time steps is given in figure 5.5(a). Apart from the small errors close to the interface during the early times due to the sharp gradients, the agreement is excellent.

The amount of mass accumulated on the interface can be found from the 1D solution via mass conservation,

$$
M_{f}(t)=M_{F}(0)-2 \pi \int_{a}^{R} F(t) r \mathrm{~d} r .
$$

Figure 5.5(b) shows that the diffuse-interface solution is in good agreement with the $1 \mathrm{D}$ solution.

The error and convergence for both the bulk concentration and the surface concentration is given in Table 5.5. The error decreases in a second-order fashion. The reason for this is that for this test case, the surface concentration is uniform, so there is no surface diffusion.

Next, the effect of varying the surface diffusion coefficient relative to the bulk diffusion coefficient, $D=D_{f} / D_{F}$, is investigated. The initial bulk concentration is set to

$$
F_{0}(y)=\left(y_{\max }-y\right) \times 10^{-3}
$$

instead of the previous uniform concentration and we consider both adsorption and desorption. There is a non-uniform adsorption to the interface which gives rise to 


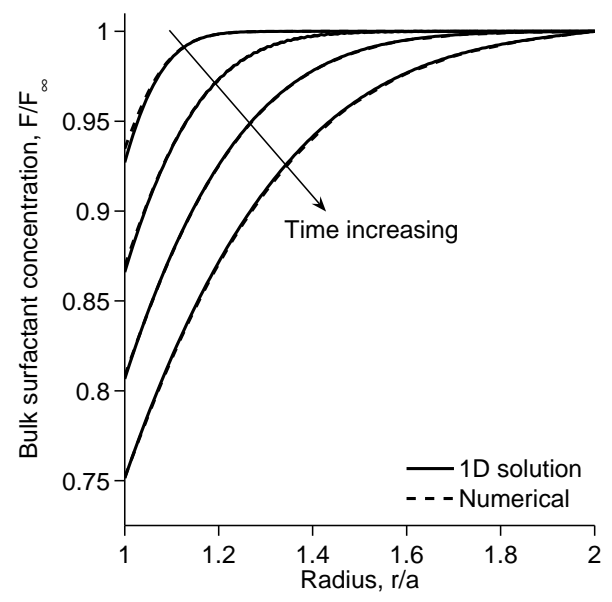

(a) The $y=0$ slice of the bulk concentration distribution at times $t=0.005,0.02,0.05$ and 0.1 .

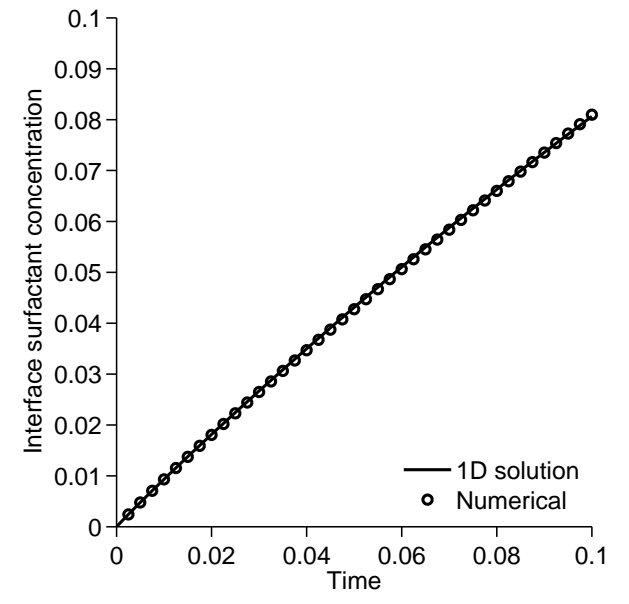

(b) Interface surfactant concentration.

Fig. 5.5: Comparison of the 4th order 1D solution and the phase-field solution for the bulk diffusion and bulk-surface mass transfer test case. $h_{\min }=1 / 128, \epsilon=1 / 80$.

Table 5.5: The error in the infinity norm and convergence order for the bulk concentration and the accumulated surface mass in the bulk diffusion and bulk-surface mass transfer test case. The interface thickness is $\epsilon=1.6 h_{\mathrm{min}}$.

\begin{tabular}{ccccc}
\hline Grid spacing & \multicolumn{2}{c}{ Bulk concentration } & \multicolumn{2}{c}{ Surface mass } \\
& $\begin{array}{c}\text { Error } \\
\left(\times 10^{-2}\right)\end{array}$ & Order & $\begin{array}{c}\text { Error } \\
\left(\times 10^{-3}\right)\end{array}$ & Order \\
\hline $1 / 8$ & 11.38 & - & 9.66 & - \\
$1 / 16$ & 5.21 & 1.13 & 4.50 & 1.10 \\
$1 / 32$ & 1.57 & 1.73 & 1.20 & 1.91 \\
$1 / 64$ & 0.343 & 2.20 & 0.22 & 2.44 \\
$1 / 128$ & 0.0473 & 2.86 & 0.036 & 2.61 \\
\hline
\end{tabular}

diffusion effects on the surface. Additionally, desorption is added to yield more complex dynamics. The radius is set to $r=0.15$ and the drop is centered at $(0.5,0.5)$. The adsorption and desorption rates are $r_{a}=r_{d}=100$ and the bulk diffusion coefficient is $D_{F}=1.0$. Figure 5.6 shows the concentration in the bulk and on the surface at $t=0.08$ for varying diffusion ratios. A grid spacing of $h=1 / 256$ was employed, along with interface thickness $\epsilon=0.004$. Because of the non-uniform bulk distribution, the adsorption will be higher at the bottom of the circle than at the top. As the surface diffusion coefficient increases, mass is diffused from the bottom to the top of the circle faster, which further reduces the adsorption at the top. This effectively increases the diffusion and leads to a more uniform concentration distribution in the bulk. This is illustrated in figure 5.7, which shows the bulk concentration along $x=0.5$. The larger surface diffusion also leads to lower total interface concentration, since mass is 

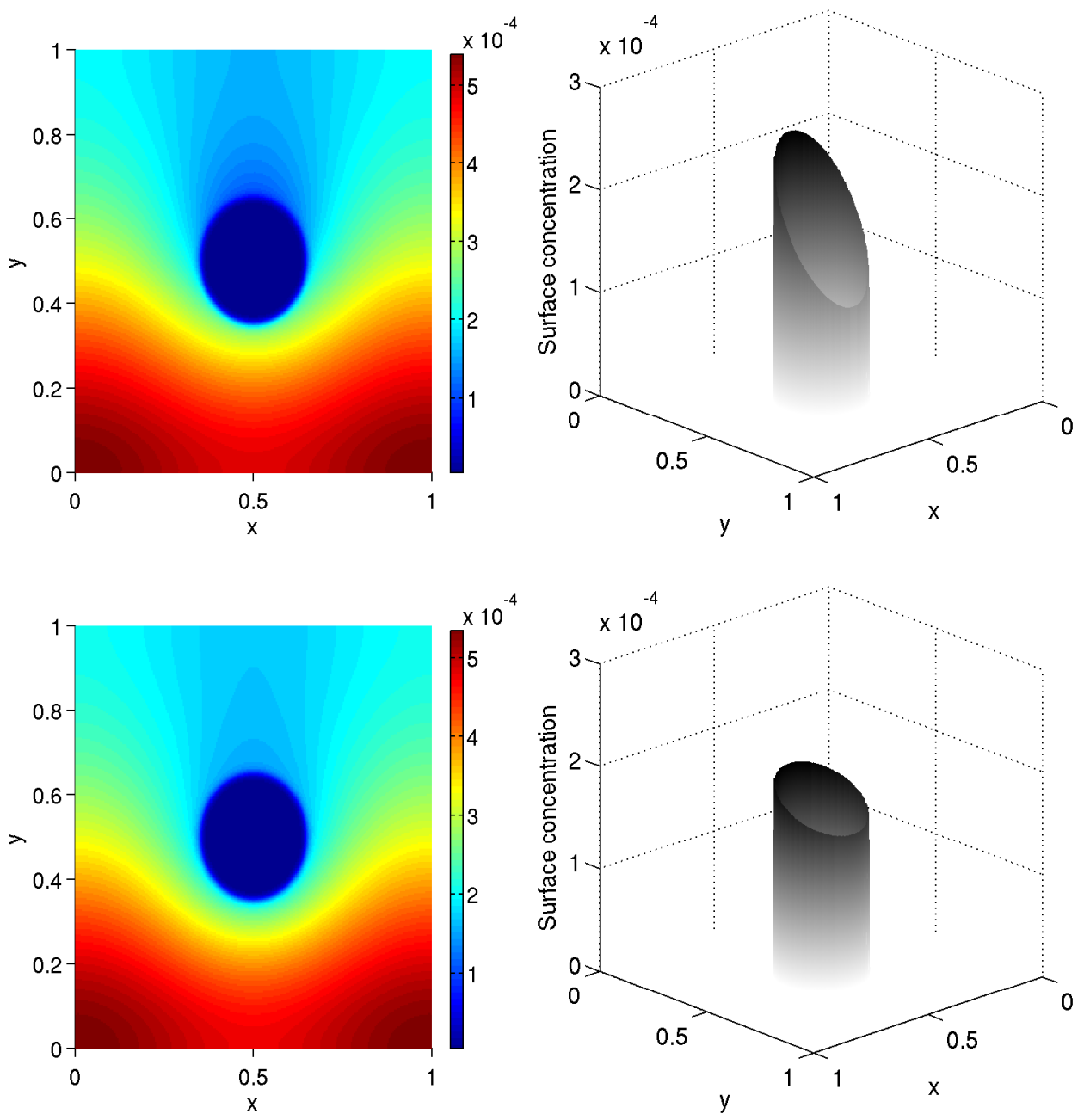

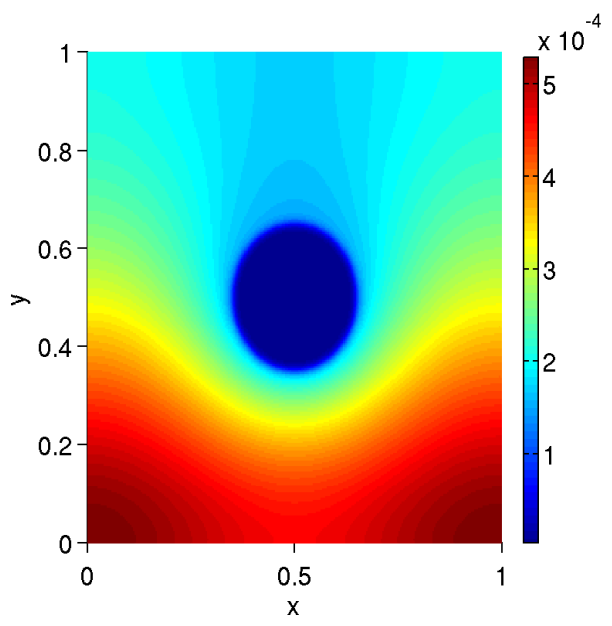

(a) Bulk concentration

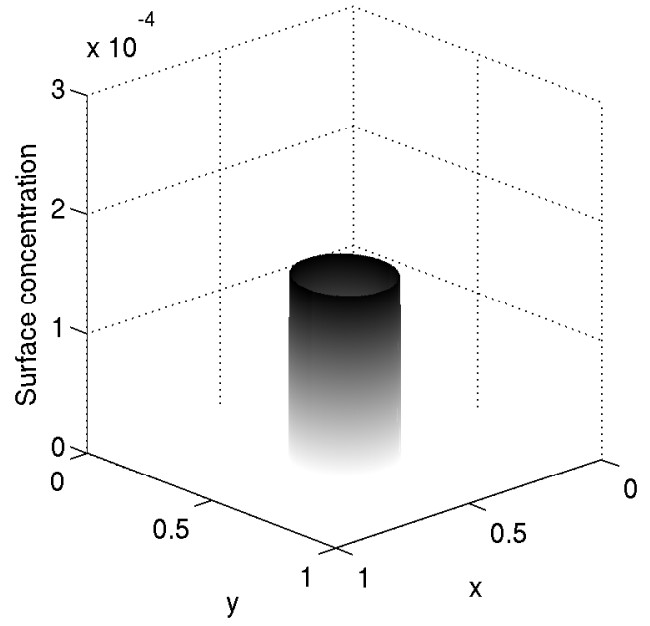

(b) Surface concentration

Fig. 5.6: Bulk concentration and surface concentration for the mass transfer test case with non-uniform initial bulk concentration at $t=0.08$. From top to bottom, $D=0.1$, $D=1.0$ and $D=10$. 


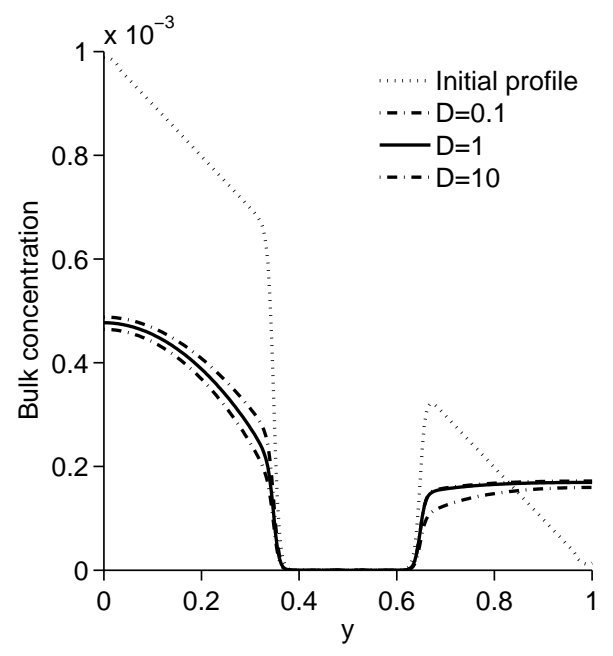

Fig. 5.7: Bulk concentration along $x=0.5$ for three different diffusion coefficient ratios at $t=0.08$. A larger surface diffusion coefficient increases the transport of bulk concentration and gives a more uniform profile.

desorbed back into the bulk.

5.5. Bulk-surface coupling on a perturbed circle In this test case, the surface evolution on, and the bulk evolution inside, a perturbed circle is considered. The circle is given by

$$
r(\theta)=1+0.1 \cos (3 \theta)
$$

and forcing functions are added to the surface and bulk equations so that the exact solution is known. More specifically,

$$
\begin{aligned}
& \frac{\partial f}{\partial t}=\nabla_{s}^{2} f-f+F+\zeta_{1} \text { on } \Gamma \\
& \frac{\partial F}{\partial t}=\nabla^{2} F-F+\zeta_{2} \text { in } \Omega_{0}
\end{aligned}
$$

is solved subject to the boundary condition

$$
\boldsymbol{\nabla} F \cdot \mathbf{n}=f-F \text { on } \Gamma \text {. }
$$

The forcing functions are chosen such that the solution in $\Omega_{0}$ is

$$
F=\frac{1}{4}\left(x^{2}+y^{2}\right) e^{-3 t},
$$

and the solution on $\Gamma$ is

$$
f=\left(\frac{1}{2} \frac{r(\theta)}{\sqrt{\left.r(\theta)^{2}+r^{\prime}(\theta)\right)^{2}}}+\frac{1}{4} r(\theta)^{2}\right) e^{-3 t} .
$$




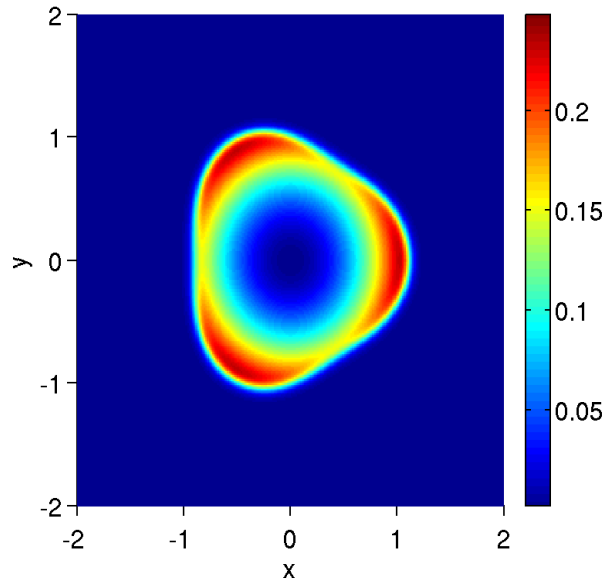

(a) Bulk concentration

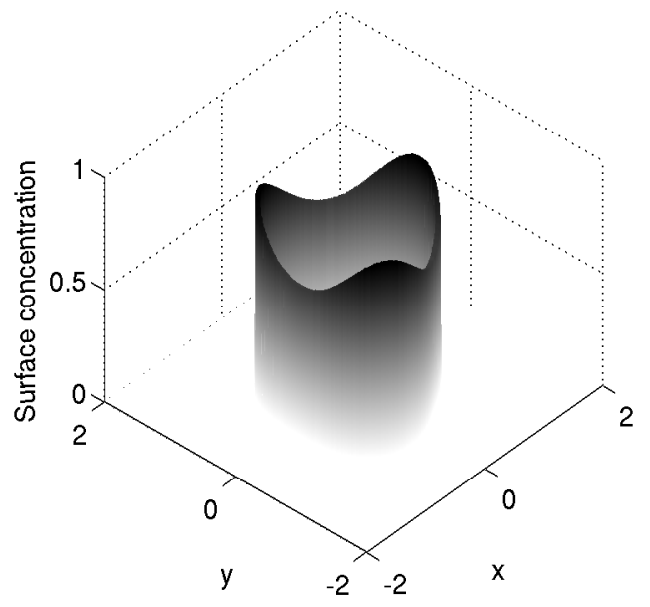

(b) Surface concentration

Fig. 5.8: The solutions at $t=2.0 \times 10^{-3}$ for the bulk-surface coupling on a perturbed circle.

The diffuse-interface representation of the above system is

$$
\begin{aligned}
& \frac{\partial}{\partial t}(f B(c))=\boldsymbol{\nabla} \cdot(B(c) \boldsymbol{\nabla} f)-B(c)\left(f+F+\zeta_{1}\right) \\
& \frac{\partial}{\partial t}(H F)=\boldsymbol{\nabla} \cdot(H \boldsymbol{\nabla} F)-H F+H \zeta_{2}+(f-F)|\boldsymbol{\nabla} c|,
\end{aligned}
$$

where the Heaviside function is now given by

$$
H(c)=1-c,
$$

instead of equation (2.17) since the bulk concentration is required in $\Omega_{0}$ (not in $\Omega_{1}$ ).

The simulation was run to time $t=2.0 \times 10^{-3}$, and the interface thickness was scaled as $\epsilon=1.28 h_{\mathrm{min}}$. Figure 5.8 shows the final solution for the surface concentration and the bulk concentration for a grid spacing of $h_{\min }=1 / 128$. The analytical solutions given by equations (5.17) and (5.18) are compared to the numerical results in figure 5.9. Excellent agreement is observed. In Table 5.6, the respective errors compared to the analytical solutions are listed together with the convergence order. Again, first order convergence results are obtained.

5.6. Bulk-surface coupling in a $2 \mathrm{D}$ vortical flow $\mathrm{A}$ circle with radius $r=0.15$ is placed at $\mathbf{x}=(0.5,0.75)$ in a domain $\Omega=[0,1] \times[0,1]$. The circle is advected by a prescribed velocity field defined by the stream function

$$
\Psi=-\cos (\pi t) \frac{1}{\pi} \sin ^{2}(\pi x) \sin ^{2}(\pi y),
$$

which gives the individual velocity components as

$$
\begin{aligned}
& u=-2 \cos (\pi t) \sin ^{2}(\pi x) \sin (\pi y) \cos (\pi y), \\
& v=2 \cos (\pi t) \sin ^{2}(\pi y) \sin (\pi x) \cos (\pi x) .
\end{aligned}
$$




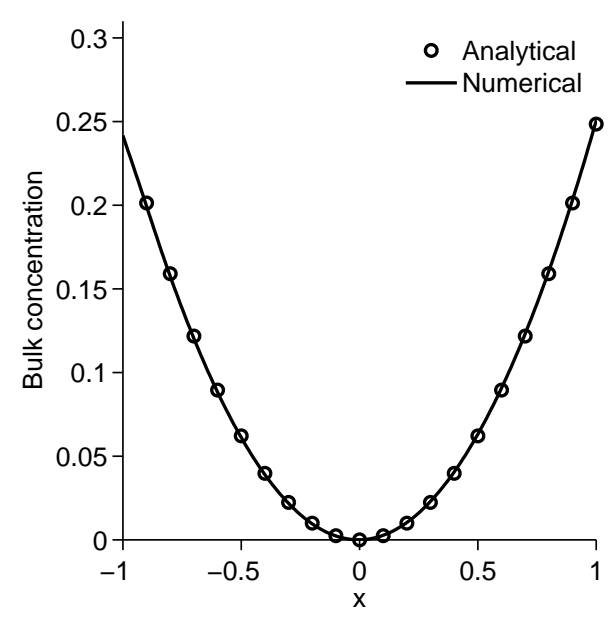

(a) Bulk concentration along $y=0$

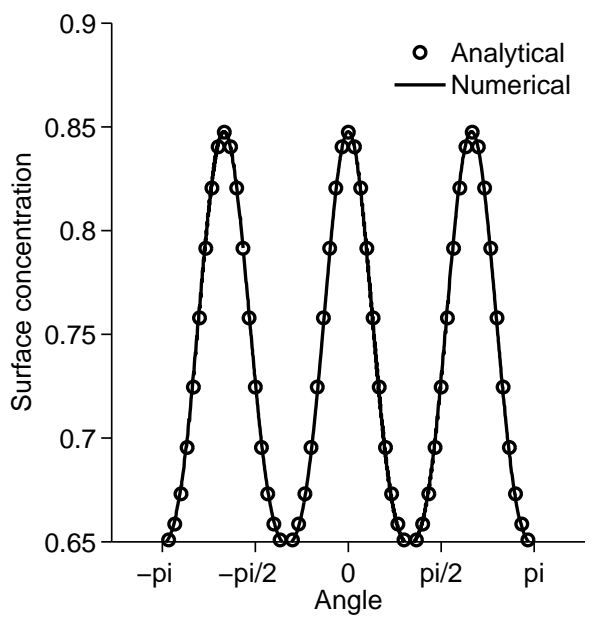

(b) Surface concentration

Fig. 5.9: Comparison between the analytical solutions and numerical solutions for $h=1 / 128$ at $t=2.0 \times 10^{-3} \mathrm{~s}$ for the perturbed circle test case.

Table 5.6: The error in the infinity norm and convergence order for the bulk concentration and the interface concentration in the perturbed circle test case. The interface thickness is $\epsilon=1.28 \mathrm{~h}$.

\begin{tabular}{ccccc}
\hline Grid spacing & \multicolumn{2}{c}{ Bulk concentration } & \multicolumn{2}{c}{ Surface concentration } \\
& $\begin{array}{c}\text { Error } \\
\left(\times 10^{-3}\right)\end{array}$ & Order & $\begin{array}{c}\text { Error } \\
\left(\times 10^{-2}\right)\end{array}$ & Order \\
\hline $1 / 16$ & 17.0 & - & 11.0 & - \\
$1 / 32$ & 9.5 & 0.84 & 4.4 & 1.32 \\
$1 / 64$ & 5.1 & 0.90 & 1.5 & 1.55 \\
$1 / 128$ & 2.6 & 0.97 & 0.74 & 1.02 \\
\hline
\end{tabular}

The initial condition is illustrated in figure 5.10. This is a demanding test case for both the interface advection and the adaptive grid, because the interface undergoes large deformations. Because of the periodicity of the flow field, it is common to compare the initial solution to the solution after one period. However, with diffusion and adsorption/desorption, the solution is not time-reversible. Here, a comparison with the results from [90] is first considered as a validation case, before simulations with bulk/interface coupling are presented.

For the validation case, the diffusion is set to $D_{f}=10^{-3}$ for the surface concentration, and the evolution of the bulk concentration is not considered. Hence, there is no coupling between the bulk and surface for this test case. The initial surface concentration is $f_{0}=1.0$. An effective grid spacing of $h_{\min }=1 / 512$ was used along with an interface thickness $\epsilon=0.002$. Figure 5.11(a) shows the evolution of the interface and the adaptive grid. The grid clearly tracks the interface in an efficient manner, and the 


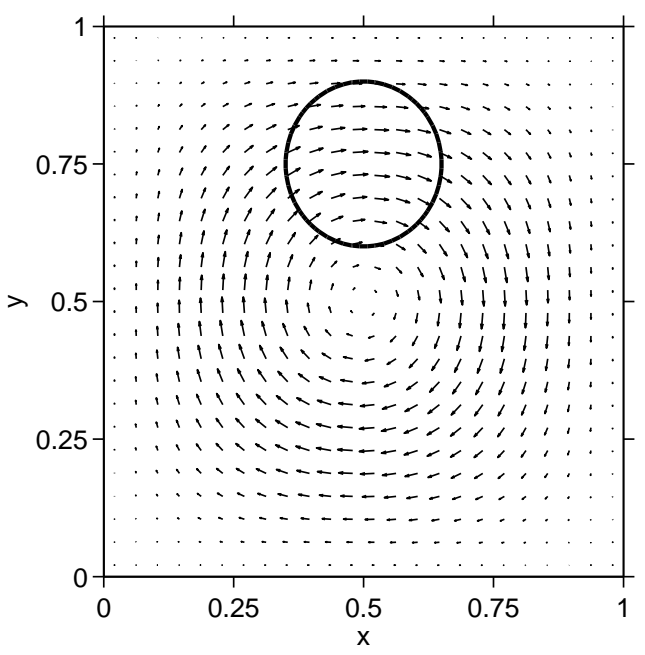

Fig. 5.10: Illustration of the vortical velocity field and initial surface position.

morphology at $t=0.5$ matches that of figure 11(b) in [90]. The surface concentration along the interface is shown in figure 5.11(b). Due to the complex flow field, mass is swept to the lower and upper part of the stretching circle. The concentration is particularly high at the upper part. This profile is in good agreement with the equivalent figure $11(\mathrm{c})$ in [90].

Next, the effect of varying the surface diffusion coefficient relative to the bulk diffusion coefficient, $D=D_{f} / D_{F}$, is investigated. The initial surface concentration is $f_{0}=10^{-4}$ and the initial bulk concentration is given by the non-uniform profile $F_{0}=10^{-4} y$. Additionally, the bulk and interface is coupled, with adsorption coefficient $r_{a}=100$ and desorption coefficient $r_{d}=200$. The bulk diffusion coefficient is $D_{F}=0.1$, while the surface diffusion coefficient is varied. An effective grid spacing of $h_{\min }=1 / 256$ was employed, along with interface thickness $\epsilon=0.004$.

The concentration at $t=0.5$ for the bulk and the interface for three different diffusion coefficient ratios is shown in figure 5.12. When the surface diffusion coefficient is low, the main transport mechanism is the convection. Some mass is adsorbed onto the interface, but the low diffusion coefficient leads to a highly non-uniform profile. As the surface diffusion coefficient increases, more mass is adsorbed from the upper part of the domain, where the bulk concentration is high, then diffused along the interface and finally desorbed in the lower part of the domain. This leads to a more uniform bulk distribution around the advected circle.

Finally, a test of the efficiency of the multigrid algorithm and the adaptive grid was performed on this test case. This was done by comparing the time taken to complete 1000 time steps on several grid sizes on both uniform and adaptive grids. The time step size was kept constant, and low enough so that the number of V-cycles on each time step was equivalent for all grid sizes. The results are shown in figure 5.13. Both the uniform and adaptive run-times increase in a linear fashion, which indicates that the numerical implementation achieves the optimal $\mathcal{O}(N)$ run-time complexity of the multigrid algorithm, where $N$ is the total number of grid points. Additionally, the slope for the adaptive simulations is much lower than the corresponding slope for the 

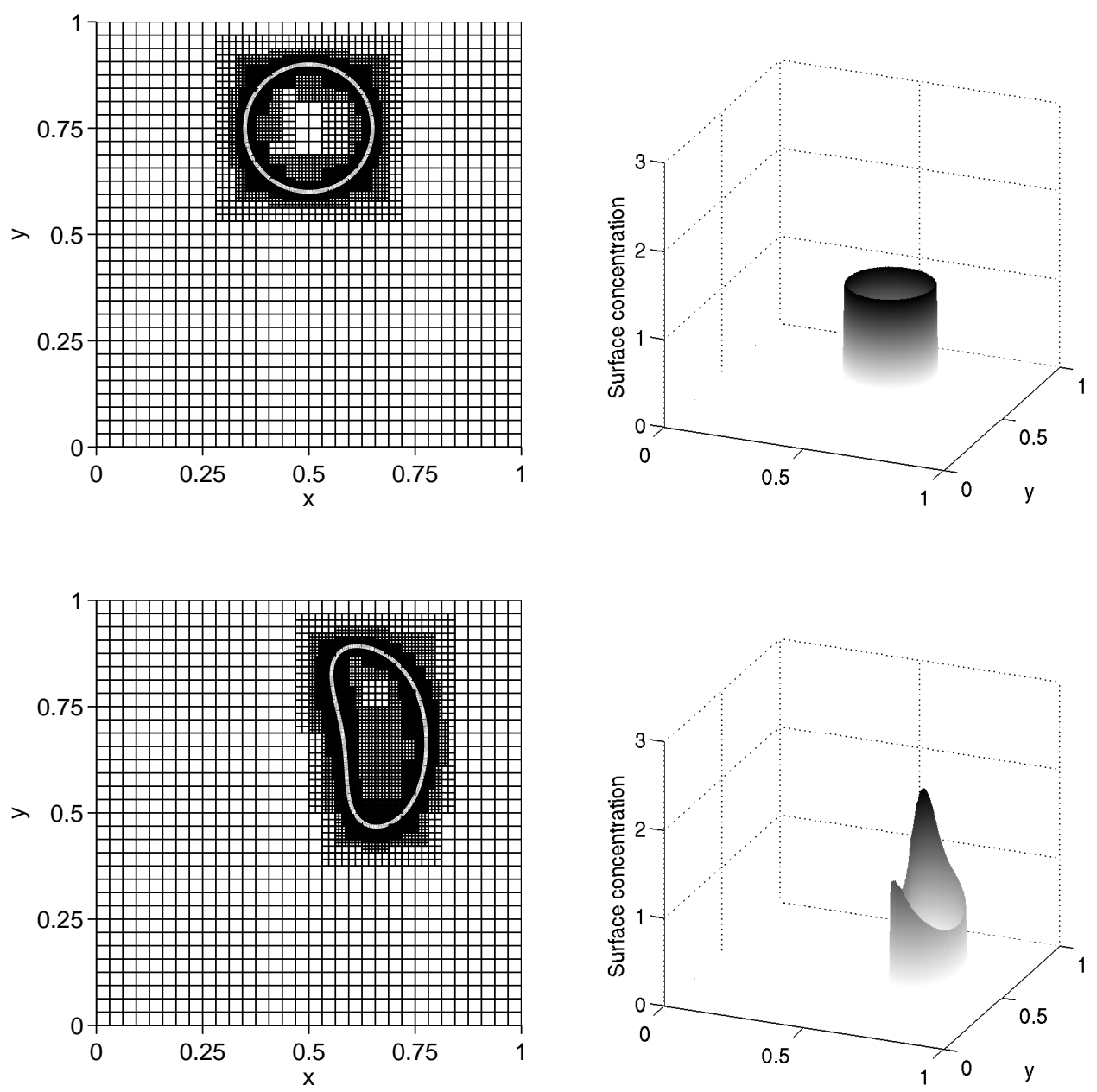

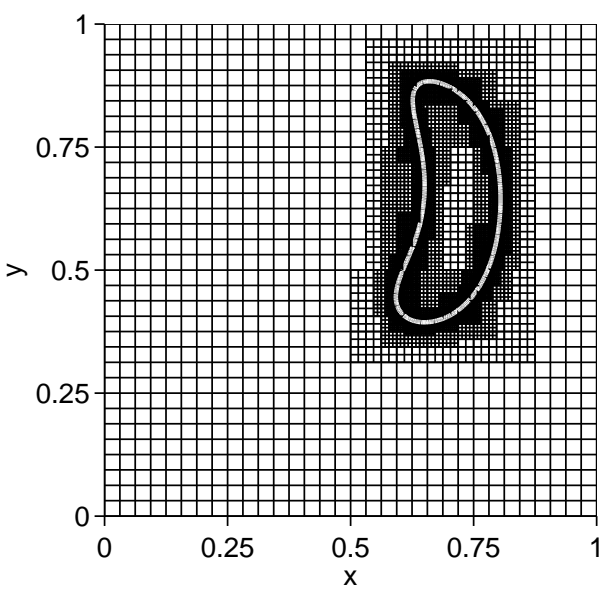

(a) Adaptive grid and interface morphology.

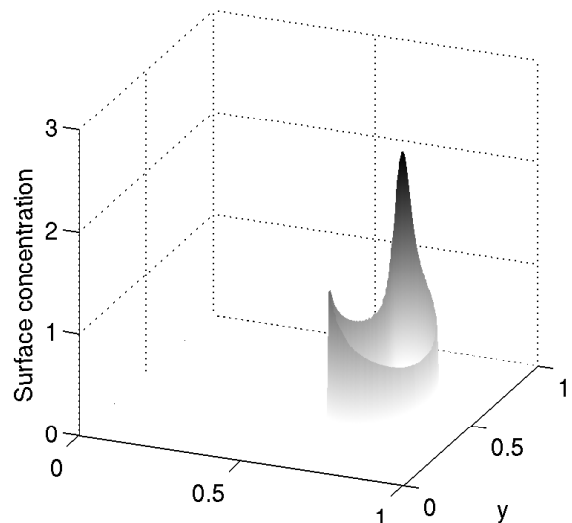

(b) Surface concentration along the interface.

Fig. 5.11: Evolution of a circle in a vortex field, depicted at $t=0.0,0.25,0.5$. The parent grid level has spacing $h=1 / 32$, and there are 4 levels of refinement, giving an effective grid spacing of $h_{\min }=1 / 512$. 

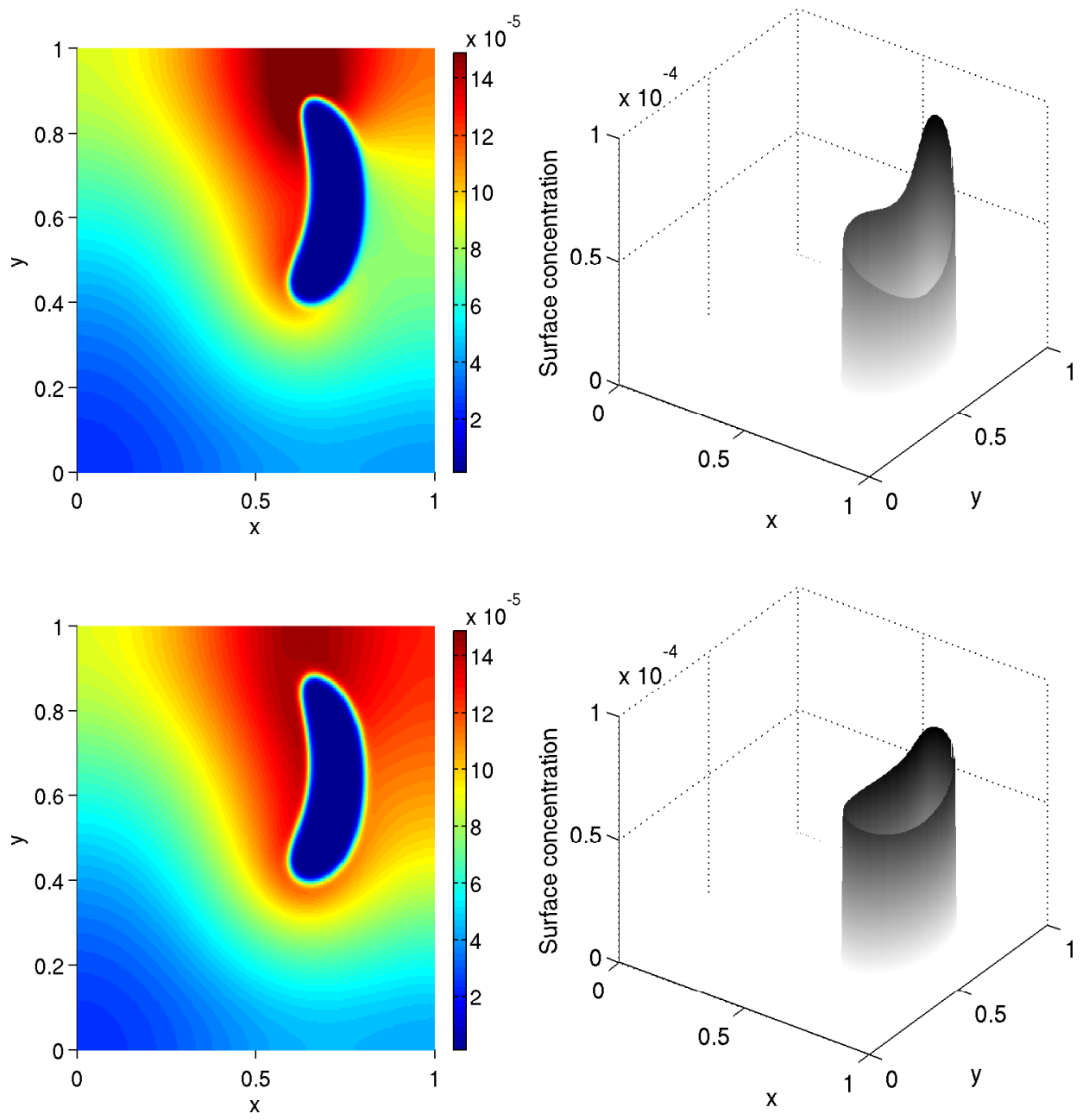

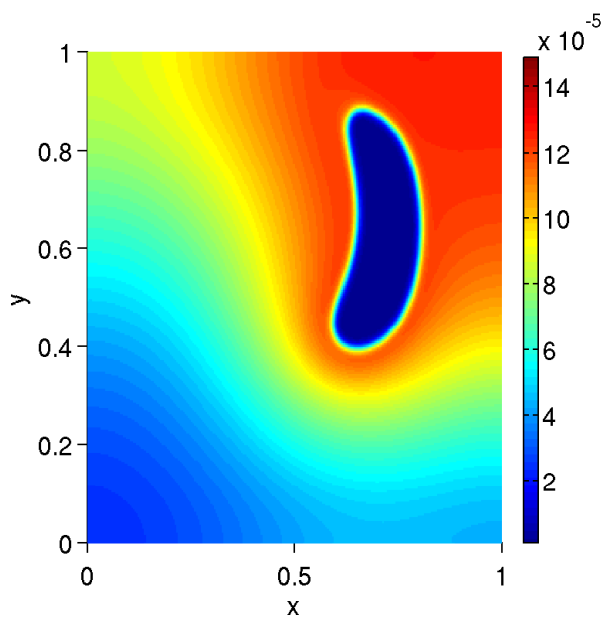

(a) Bulk concentration

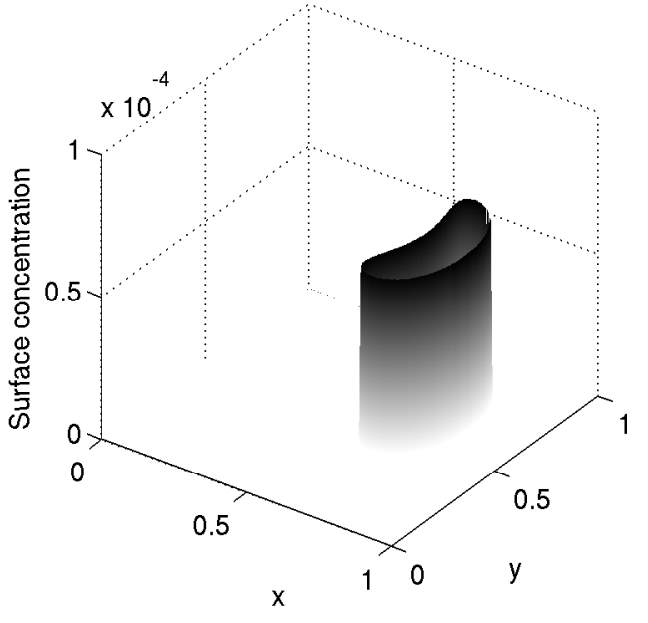

(b) Surface concentration

Fig. 5.12: Bulk concentration and interface concentration at $t=0.5$ for the vortex test case with non-uniform initial bulk concentration and bulk/interface coupling. From top to bottom, $D=0.1, D=1.0$ and $D=10$. 


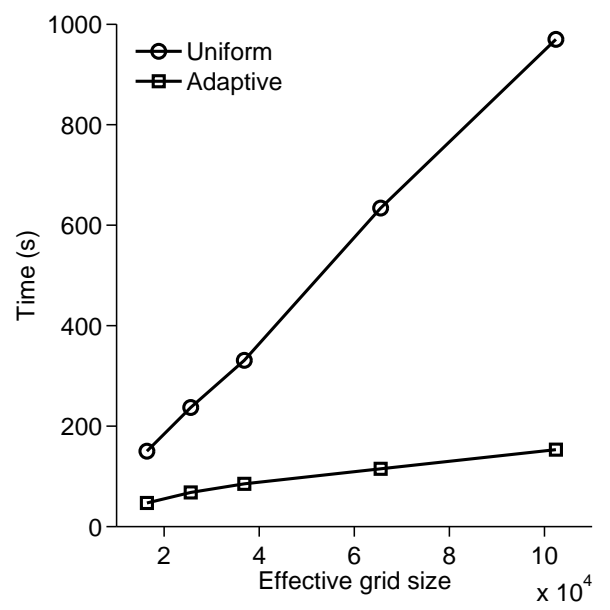

Fig. 5.13: Run-time of the vortex simulation versus grid size for both uniform and adaptive grids.

uniform simulations, which is a demonstration of the efficiency of block-structured, adaptive grids for these types of simulations.

5.7. Bulk-surface coupling in a $3 \mathrm{D}$ deformation field Finally, a threedimensional test problem is considered. The flow field is the one proposed by [50], which combines a deformation in the $x-y$ plane with one in the $x$ - $z$ plane. The velocity components are

$$
\begin{aligned}
& u=2 \cos \left(\frac{\pi t}{3}\right) \sin ^{2}(\pi x) \sin (2 \pi y) \sin (2 \pi z), \\
& v=-\cos \left(\frac{\pi t}{3}\right) \sin (2 \pi x) \sin ^{2}(\pi y) \sin (2 \pi z), \\
& w=-\cos \left(\frac{\pi t}{3}\right) \sin (2 \pi x) \sin (2 \pi y) \sin ^{2}(\pi z),
\end{aligned}
$$

and a sphere of radius 0.15 is placed at $(0.35,0.35,0.35)$ in a unit cube computational domain. The initial surface concentration is $f_{0}=10^{-4}$ and the initial bulk concentration is given by the non-uniform profile $F_{0}=10^{-4} y$. Additionally, the bulk and interface is coupled, with adsorption coefficient $r_{a}=100$ and desorption coefficient $r_{d}=200$. The bulk and surface diffusion coefficients are matched with $D_{F}=D_{f}=1$. A grid with spacing $h=1 / 32$ at the root level and three levels of refinement is used $\left(h_{\min }=1 / 256\right)$, along with the interface thickness $\epsilon=0.004$.

Figure 5.14(a) shows the evolution of the sphere. The morphology agrees well with figure 29 from [18], where the same problem was solved with the level set method, only without any surface quantities. As the interface stretches, the surface concentration becomes lower. The concentration is higher at the front of the deforming sphere, because of adsorption from the bulk. The bulk concentration increases in the $y$ direction, and the front of the sphere moves through this part, thereby increasing the local concentration on the front. This can be seen from the slice of the bulk concentration, figure 5.14(b), at time 1.0, where the concentration is lower near the 
region that the interface has moved through. The middle of the stretched sphere has a much lower concentration, due to the fact that it has experienced a large deformation and is in a region of low bulk concentration.

Figure 5.15 shows a sequence of the block-structured grids used in the simulation. The boxes denote grid level boundaries, so that inside each box the resolution is doubled. This demonstrates that the adaptive grid algorithm also works well for three-dimensional problems.

6. Conclusion A diffuse-interface method for solving problems involving transport, diffusion, and adsorption/desorption of a material quantity on a deformable interface was presented. The method was shown to perform well on a wide range of test cases. The efficiency of the numerical implementation, using adaptive grids and a multigrid method, was also demonstrated.

The asymptotic analysis suggests, and numerical evidence confirms, that the convergence to the sharp interface system is first order in the interface thickness parameter $\epsilon$. It may be possible to gain second order accuracy in $\epsilon$ by explicitly removing the corresponding term in the asymptotic expansion as can be done in the context of solidification to enable simulations with arbitrary kinetic coefficients [43, 44]. This should be explored.

A natural extension of the method is to couple it to an external flow solver. The ability of the diffuse-interface method to handle complex fluids and interfacial dynamics makes this a very attractive combination. We are currently developing such an algorithm to simulate the dynamics of interfacial flows with soluble surfactants. Another interesting extension is to couple the method with models of cellular mechanics to simulate cell-polarization and motility.

Finally, this work used a phase-field function to represent the interface. Alternatively, a level-set function could be used instead. Accurate representations of delta functions and Heaviside functions in the level-set context can be found in for example $[80,83,68]$.

Acknowledgment. The authors thank Steven Wise and Fang Jin for assistance with the numerical code and visualization, and the reviewers, whose comments have improved the paper. KET is funded by the project "Electrocoalescence - Criteria for an efficient process in real crude oil systems"; coordinated by SINTEF Energy Research. The project is supported by The Research Council of Norway, under the contract no: 169466/S30, and by the following industrial partners: Aibel AS, Aker Solutions AS, BP Exploration Operating Company Ltd, Saudi Aramco, Shell Technology Norway AS, StatoilHydro ASA and Petrobras. KET also acknowledges support from the Research Council of Norway through grant IS-BILAT 192532 and from the Fulbright Foundation. FW, JL and XL acknowledge support from the National Science Foundation Division of Mathematical Sciences (DMS) and from the National Institutes of Health through grant P50GM76516 for a Centre of Excellence in Systems Biology at the University of California, Irvine. AV acknowledges support from the German Science Foundation through grants Vo899/6-1 and SFB 609. 

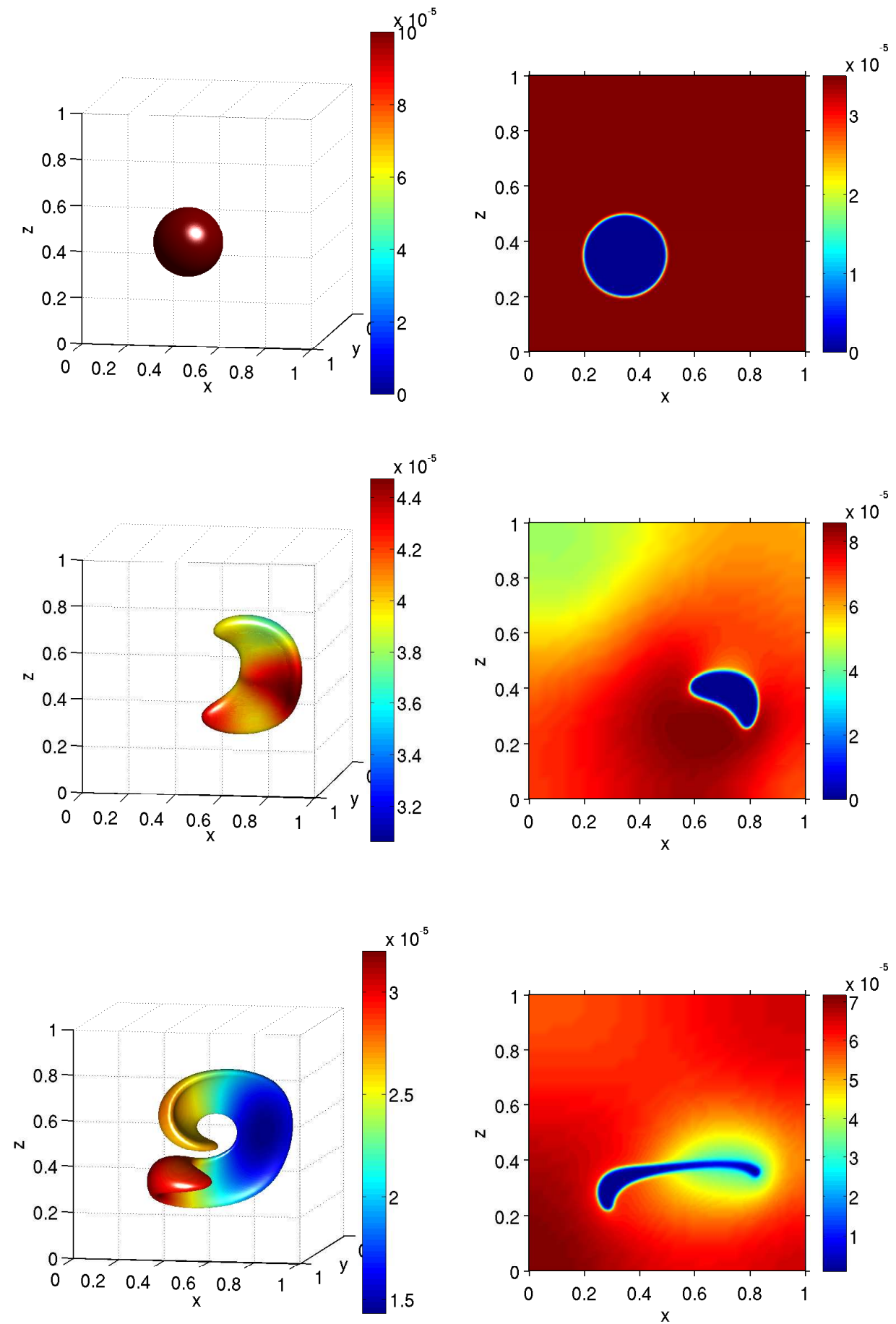

(a) Surface concentration

(b) Slice of bulk concentration

Fig. 5.14: Sphere in 3D deformation field. The left column shows the interface and the surface concentration at times $0,0.5$ and 1.0. The right column shows slices of the bulk concentration at the same times and $y$-positions $0.35,0.5$ and 0.6 , respectively. 

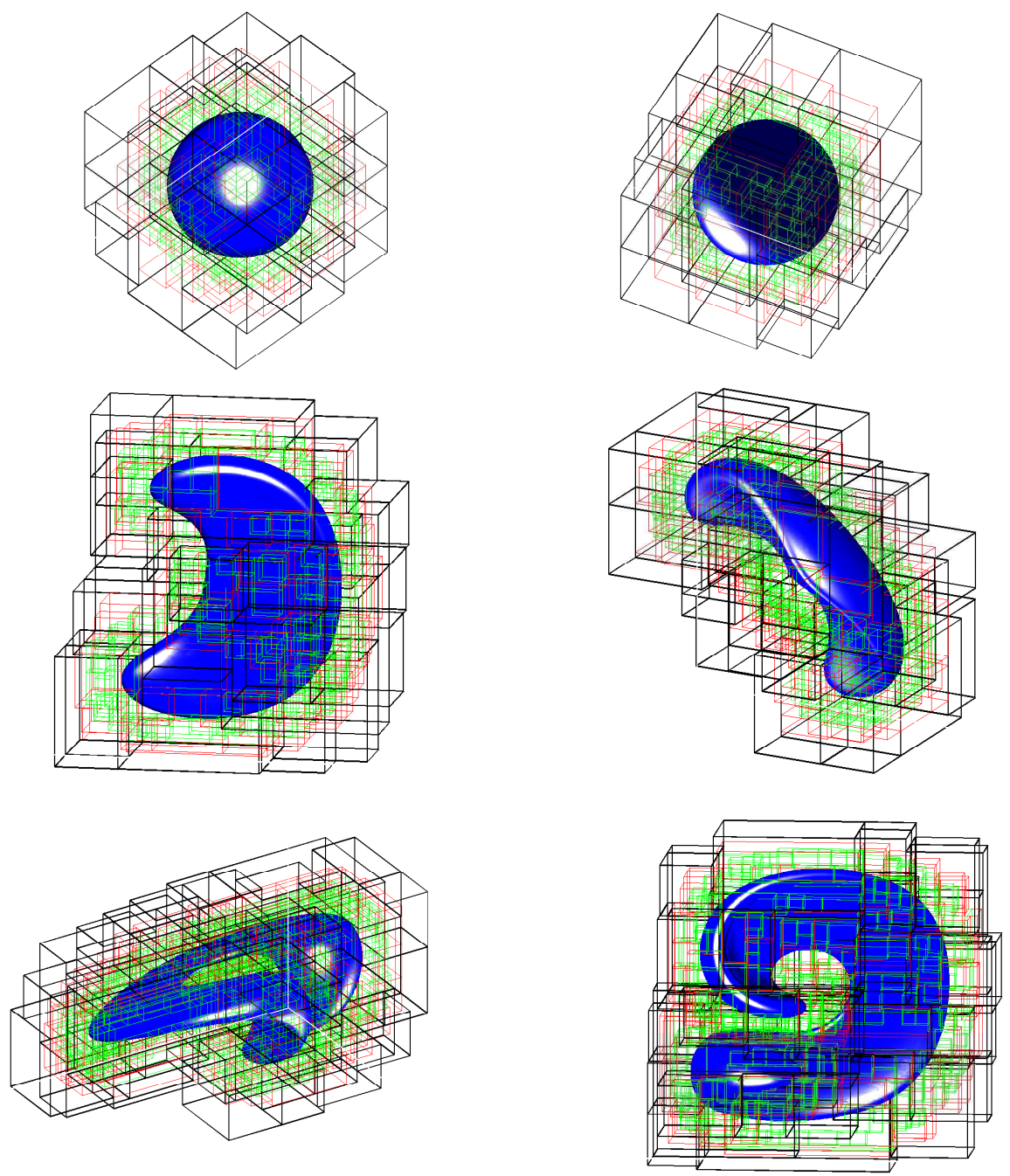

Fig. 5.15: Sphere in 3D deformation field. Illustration of the adaptive grids at times (from top to bottom) $0,0.5$ and 1.0. Two different angles are shown for each time step. Inside the black boxes, the grid spacing is $h=1 / 64$, inside the red boxes $h=1 / 128$ and inside the green boxes $h=h_{\min }=1 / 256$.

\section{REFERENCES}

[1] D. Adalsteinsson and J. A. Sethian, The fast construction of extension velocities in level set methods, J. Comput. Phys., 148, 2-22, 1999.

[2] J.-M. Allain and M. B. Amar, Biphasic vesicle: instability induced by adsorption of proteins, Physica A, 337, 531-545, 2008.

[3] D. M. Anderson, G. B. McFadden, and A. A. Wheeler, Diffuse-interface methods in fluid mechanics, Ann. Rev. Fluid Mech., 30, 139-165, 1998.

[4] J. Barrett, J. Blowey, and H. Garcke, Finite element approximation of the Cahn-Hilliard equation with degenerate mobility, SIAM J. Numer. Anal., 37, 286-318, 1999.

[5] M. Bertalmio, L. Cheng, S. Osher, and G. Sapiro, Variational problems and partial differential 
equations on implicit surfaces, J. Comput. Phys., 174, 759-780, 2001.

[6] J. Blowey and C. Elliott, The Cahn-Hilliard gradient theory for phase separation with nonsmooth free energy part 1: mathematical analysis, Eur. J. Appl. Math., 2, 233-280, 1991.

[7] J. Blowey and C. Elliott, The Cahn-Hilliard gradient theory for phase separation with nonsmooth free energy part 2: Numerical analysis, Eur. J. Appl. Math., 3, 147-179, 1992.

[8] A. Bueno-Orovio and V. Perez-Garcia, Spectral methods for partial differential equations on irregular domains: the spectral smoothed boundary method, SIAM J. Sci. Comput., 28, 886-900, 2006.

[9] A. Bueno-Orovio and V. Perez-Garcia, Spectral smoothed boundary methods: the role of external boundary conditions, Numer. Meth. Partial Diff. Eqns., 22, 435-448, 2006.

[10] G. Caginalp and P. Fife, Dynamics of layered interfaces arising from phase boundaries, SIAM J. Appl. Math., 48, 506-518, 1988.

[11] P. Cermelli, E. Fried, and M. Gurtin, Transport relations for surface integrals arising in the formulation of balance laws for evolving fluid interfaces, J. Fluid Mech., 544, 339-351, 2005.

[12] C. Duarte, I. Babuska, and J. Oden, Generalized finite element methods for three-dimensional structural mechanics problems, Comp. Struct., 77, 215-232, 2000.

[13] G. Dziuk and C. Elliott, Eulerian finite element method for parabolic PDEs on complex surfaces, Int. Free Bound., 10, 119-138, 2008.

[14] G. Dziuk and C. Elliott, An Eulerian approach to transport and diffusion on evolving implicit surfaces, Comput. Visualization Sci., in press, 2009.

[15] C. Eilks and C. Elliott, Numerical simulation of dealloying by surface dissolution by the evolving surface finite element method, J. Comput. Phys., 227, 9727-9741, 2008.

[16] C. Elliott and B. Stinner, Analysis of a diffuse interface approach to an advection diffusion equation on a moving surface, Math. Mod. Meth. Appl. Sci., in press, 2009.

[17] C. Elliott, B. Stinner, V. Styles, and R. Welford, Numerical computation of advection and diffusion on evolving diffuse interfaces, preprint, 2009.

[18] D. Enright, R. Fedkiw, J. Ferziger, and I. Mitchell, A hybrid particle level set method for improved interface capturing, J. Comput. Phys., 183, 83-116, 2002.

[19] J. Erlebacher, M. Aziz, A. Karma, N. Dimitrov, and K. Sieradzki, Evolution of nanoporosity in dealloying, Nature, 410, 450-453, 2001.

[20] R. Fedkiw, T. Aslam, B. Merriman, and S. Osher, A non-oscillatory Eulerian approach to interfaces in multimaterial flows (the ghost fluid method), J. Comput. Phys, 152, 457-492, 1999.

[21] F. Fenton, E. Cherry, A. Karma, and W.-J. Rappel, Modeling wave propagation in realistic heart geometries using the phase-field method, Chaos, 15, 103502, 2005.

[22] P. Fife and O. Penrose, Interfacial dynamics for thermodynamically consistent phase-field models with nonconserved order parameter, Elect. J. Diff. Eqs., 16, 1-49, 1995.

[23] E. Fried and M. Gurtin, A unified treatment of evolving interfaces accounting for small deformations and atomic transport with emphasis on grain-boundaries and epitaxy, Adv. Appl. Mech., 40, 1-177, 2004.

[24] F. Gibou and R. Fedkiw, A fourth order accurate discretization for the Laplace and heat equations on arbitrary domains with applications to the Stefan problem, J. Comput. Phys, 202, 577-601, 2005.

[25] F. Gibou, R. Fedkiw, L. Cheng, and M. Kang, A second order accurate symetric discretization of the Poisson equation on irregular domains, J. Comput. Phys, 176, 205-227, 2002.

[26] J. Glimm, D. Marchesin, and O. McBryan, A numerical method for 2 phase flow with an unstable interface, J. Comput. Phys, 39, 179-200, 1981.

[27] R. Glowinski, T. Pan, and J. Periaux, A fictitious domain method for external incompressible viscous-flow modeled by Navier-Stokes equations, Comput. Meth. Appl. Mech. Engin., 112, 133-148, 1994.

[28] R. Glowinski, T. Pan, R. Wells, and X. Zhou, Wavelet and finite element solutions for the Neumann problem using fictitious domains, J. Comput. Phys., 126, 40-51, 1996.

[29] A. Gomez-Marin, J. Garcia-Ojalvo, and J. Sancho, Self-sustained spatiotemporal oscillations induced by membrane-bulk coupling, Phys. Rev. Lett., 98, 168303, 2007.

[30] J. Greer, A. Bertozzi, and G. Sapiro, Fourth order partial differential equations on general geometries, J. Comput. Phys., 216, 216-246, 2006.

[31] W. Hackbusch and S. Sauter, Composite finite elements for the approximation of PDEs on domains with complicated micro-structures, Num. Math., 75, 447-472, 1997.

[32] M. Hameed, M. Siegel, Y.-N. Young, J. Li, M. R. Booty, and D. T. Papageorgiou, Influence of insoluble surfactant on the deformation and breakup of a bubble or thread in a viscous fluid, J. of Fluid Mech., 594, 307-340, 2008.

[33] J. Hao, T. Pan, R. Glowinski, and D. Joseph, A fictitious domain/distributed lagrange multiplier 
method for the particulate flow of Oldroyd-B fluids: a positive definiteness preserving approach, J. Non-Newtonian Fluid Mech., 156, 95-111, 2009.

[34] A. Hirsa and W. W. Willmarth, Measurements of vortex pair interaction with a clean or contaminated free surface, J. Fluid Mech., 259, 25-45, 1994.

[35] Y. T. Hu, D. J. Pine, and L. G. Leal, Drop deformation, breakup, and coalescence with compatibilizer, Phys. Fluids, 12, 484-489, 2000.

[36] S. D. Hudson, A. M. Jamieson, and B. E. Burkhart, The effect of surfactant on the efficiency of shear-induced drop coalescence, J. Colloid and Interface Science, 265, 409-421, 2003.

[37] K. Ito, M.-C. Lai, and Z. Li, A well-conditioned augmented system for solving Navier-Stokes in irregular domains, J. Comput. Phys., 228, 2616-2628, 2009.

[38] A. J. James and J. Lowengrub, A surfactant-conserving volume-of-fluid method for interfacial flows with insoluble surfactant, J. Comput. Phys., 201, 685-722, 2004.

[39] Y. J. Jan, Computational Studies of Bubble Dynamics, PhD thesis, University of Michigan, 1994.

[40] H. Ji, F.-S. Lien, and E. Yee, An efficient second-order accurate cut-cell method for solving the variable coefficient Poisson equation with jump conditions on irregular domains, Int. J. Num. Meth. Fluids, 52, 723-748, 2006.

[41] H. Johansen and P. Colella, A Cartesian grid embedded boundary method for Poisson's equation on irregular domains, J. Comput. Phys, 147, 60-85, 1998.

[42] H. Johansen and P. Colella, Embedded boundary algorithms and software for partial differential equations, J. Phys., 125, 012084, 2008.

[43] A. Karma and W.-J. Rappel, Phase-field method for computationally efficient modeling of solidification with arbitrary interface kinetics, Phys. Rev. E, 53, R3017-R3020, 1996.

[44] A. Karma and W.-J. Rappel, Quantitative phase-field modeling of dendritic growth in two and three dimensions, Phys. Rev. E, 57, 4323-4349, 1998.

[45] J.-S. Kim, K. Kang, and J. Lowengrub, Conservative multigrid methods for Cahn-Hilliard fluids, J. Comput. Phys., 193, 511-543, 2004.

[46] J. Kockelkoren, H. Levine, and W.-J. Rappel, Computational approach for modeling intra- and extracellular dynamics, Phys. Rev. E, 68, 037702, 2003.

[47] M.-C. Lai, Y.-H. Tseng, and H. Huang, An immersed boundary method for interfacial flows with insoluble surfactant, J. Comput. Phys., 227, 7270-7293, 2008.

[48] L. G. Leal, Flow induced coalescence of drops in a viscous fluid, Phys. of Fluids, 16, 1833-1851, 2004.

[49] R. LeVeque and Z. Li, The immersed interface method for elliptic equations with discontinuous coefficients and singular sources, SIAM J. Num. Anal., 31, 1019-1044, 1997.

[50] R. J. Leveque, High-resolution conservative algorithms for advection in incompressible flow, SIAM J Numer. Anal., 33, 627-665, 1996.

[51] H. Levine and W.-J. Rappel, Membrane-bound Turing patterns, Phys. Rev. E, 72, 061912, 2005.

[52] B. Li, J. Lowengrub, A. Ratz, and A. Voigt, Geometric evolution laws for thin crystalline films: modeling and numerics, Commun. Comput. Phys., 6, 433-482, 2009.

[53] X. Li, J. Lowengrub, A. Ratz, and A. Voigt, Solving PDEs in complex geometries: a diffuse domain approach, Comm. Math. Sci., 7, 81-107, 2009.

[54] Z. Li and K. Ito, The immersed interface method: Numerical solutions of PDEs involving interfaces and irregular domains, SIAM Front. Appl. Math., 33, 2006.

[55] S. Liu and T. Chan, Weighted essentially non-oscillatory schemes, J. Comput. Phys., 115, 200-212, 1994.

[56] R. Lohner, J. Cebral, F. Camelli, J. Baum, E. Mestreau, and O. Soto, Adaptive embedded/immersed unstructured grid techniques, Arch. Comput. Meth. Eng., 14, 279-301, 2007.

[57] W. E. Lorensen and H. E. Cline, Marching cubes: a high resolution $3 d$ surface construction algorithm, Computer Graphics, 21, 163-169, 1987.

[58] J. Lowengrub and L. Truskinovsky, Quasi-incompressible Cahn-Hilliard fluids and topological transitions, R. Soc. Lond. Proc. Ser. A Math. Phys. Eng. Sci., 454, 2617-2654, 1998.

[59] S. Lui, Spectral domain embedding for elliptic PDEs in complex domains, J. Comput. Appl. Math., 225, 541-557, 2009.

[60] P. Macklin and J. Lowengrub, Evolving interfaces via gradients of geometry-dependent interior poisson problems: application to tumor growth, J. Comput. Phys, 203, 191-220, 2005.

[61] P. Macklin and J. Lowengrub, A new ghost cell/level set method for moving boundary problems: Application to tumor growth, J. Sci. Comput., 35, 266-299, 2008.

[62] S. Marella, S. Krishnan, and H. Udaykumar, Sharp interface Cartesian grid method I: an easily implemented technique for 3D moving boundary computations, J. Comput. Phys, 210, 1-31, 2005.

[63] O. K. Matar and S. M. Troian, The development of transient fingering patterns during the 
spreading of surfactant coated films, Phys. Fluids, 11, 3232-3246, 1999.

[64] P. McCorquodale, P. Colella, and H. Johansen, A Cartesian grid embedded boundary method for the heat equation on irregular domains, J. Comput. Phys, 173, 620-635, 2001.

[65] J. Melenk and I. Babuska, The partition of unity finite element method: basic theory and applications, Comp. Meth. Appl. Mech. Eng., 139, 289-314, 1996.

[66] W. J. Milliken and L. G. Leal, The influence of surfactant on the deformation and breakup of a viscous drop - the effect of surfactant solubility, J. Colloid and Interface Sci., 166, 275-285, 1994.

[67] W. J. Milliken, H. A. Stone, and L. G. Leal, The effect of surfactant on transient motion of Newtonian drops, Phys. Fluids A, 5, 69-79, 1993.

[68] C. Min and F. Gibou, Robust second-order accurate discretizations of the multi-dimensional Heaviside and Dirac delta functions, J. Comput. Phys., 227, 9686-9695, 2008.

[69] M. Muradoglu and G. Tryggvason, A front-tracking method for computation of interfacial flows with soluble surfactants, J. Comput. Phys., 227, 2238-2262, 2008.

[70] J. Oden, C. Duarte, and O. Zienkiewicz, A new cloud-based hp finite element method, Comp. Meth. Appl. Mech. Eng., 153, 117-126, 1998.

[71] R. Pego, Front migration in the nonlinear Cahn-Hilliard equation, Proc. Roy. Soc. London A, 422, 261-278, 1989.

[72] I. Ramiere, P. Angot, and M. Belliard, A general fictitious domain method with immersed jumps and multilevel nested structured meshes, J. Comput. Phys., 225, 1347-1387, 2007.

[73] A. Ratz and A. Voigt, PDEs on surfaces - a diffuse interface approach, Commun. Math. Sci., 4, 575-590, 2006

[74] M. Rech, S. Sauter, and A. Smolianski, Two-scale composite finite element method for Dirichlet problems on complicated domains, Num. Math., 102, 681-708, 2006.

[75] Y. Y. Renardy, M. Renardy, and V. Cristini, A new volume-of-fluid formulation for surfactants and simulations of drop deformation under shear at a low viscosity ratio, European Journal of Mechanics - B/Fluids, 21, 49-59, 2002.

[76] P. Schwartz, M. Barad, P. Colella, and T. Ligocki, A Cartesian grid embedded boundary method for the heat equation and Poisson's equation in three dimensions, J. Comput. Phys, 211, $531-550,2006$.

[77] J. Sethian and Y. Shan, Solving partial differential equations on irregular domains with moving interfaces, with applications to superconformal electrodeposition in semiconductor manufacturing, J. Comput. Phys, 227, 6411-6447, 2008.

[78] C. W. Shu and S. Osher, Efficient implementation of essentially non-oscillatory shock-capturing schemes, J. Comput. Phys., 77, 439-471, 1988.

[79] I. Singer-Loginova and H. Singer, The phase field technique for modeling multiphase materials, Rep. Prog. Phys., 71, 106501, 2008.

[80] P. Smereka, The numerical approximation of a delta function with application to level set methods, J. Comput. Phys., 211, 77-90, 2006.

[81] C. Stocker and A. Voigt, A level set approach to anisotropic surface evolution with free adatoms, SIAM J. Appl. Math, 69, 64-80, 2008.

[82] H. A. Stone and L. G. Leal, The effect of surfactants on drop deformation and breakup, J. Fluid. Mech., 220, 161-186, 1990.

[83] J. D. Towers, Two methods for discretizing a delta function supported on a level set, J. Comput. Phys, 220, 915-931, 2007.

[84] S. M. Troian, E. Herbolzheimer, and S. A. Safran, Model for the fingering instability of spreading surfactant drops, Phys. Rev. Lett., 65, 333-336, 1990.

[85] U. Trottenberg, C. Oosterlee, and A. Schller, Multigrid, London, UK: Academic Press, 2000.

[86] G. Tryggvason, J. Abdollahi-Alibeik, W. W. Willmarth, and A. Hirsa, Collision of a vortex pair with a contaminated free surface, Phys. Fluids A, 4, 1215-1229, 1992.

[87] S. Wise, J.-S. Kim, and J. Lowengrub, Solving the regularized, strongly anisotropic Cahn-Hilliard equation by an adaptive nonlinear multigrid method, J. Comput. Phys., 226, 414-446, 2007.

[88] J. J. Xu, Z. Li, J. Lowengrub, and H. Zhao, A level set method for interfacial flows with surfactant, J. Comput. Phys, 212, 590-616, 2006.

[89] J. J. Xu and H. Zhao, An Eulerian formulation for solving partial differential equations along a moving interface, J. Sci. Comp., 19, 573-594, 2003.

[90] X. Yang and A. J. James, An arbitrary Lagrangian-Eulerian (ALE) method for interfacial flows with insoluble surfactants, FDMP, 3, 65-96, 2007.

[91] J. Zhang, D. Eckmann, and P. Ayyaswamy, A front tracking method for a deformable intravascular bubble in a tube with soluble surfactant transport, J. Comput. Phys., 214, 366-396, 2006. 